hep-th/0306165

HUTP-03/A003

ITEP-TH-50/02

\title{
Three-Dimensional Quantum Gravity, Chern-Simons Theory, And The A-Polynomial
}

\author{
Sergei Gukov \\ Jefferson Physical Laboratory, Harvard University, \\ Cambridge, MA 02138, USA
}

\begin{abstract}
We study three-dimensional Chern-Simons theory with complex gauge group $S L(2, \mathbb{C})$, which has many interesting connections with three-dimensional quantum gravity and geometry of hyperbolic 3-manifolds. We show that, in the presence of a single knotted Wilson loop in an infinite-dimensional representation of the gauge group, the classical and quantum properties of such theory are described by an algebraic curve called the A-polynomial of a knot. Using this approach, we find some new and rather surprising relations between the A-polynomial, the colored Jones polynomial, and other invariants of hyperbolic 3-manifolds. These relations generalize the volume conjecture and the Melvin-MortonRozansky conjecture, and suggest an intriguing connection between the $S L(2, \mathbb{C})$ partition function and the colored Jones polynomial.
\end{abstract}

June 2003 


\section{Introduction and Motivation}

In this paper we study three-dimensional Chern-Simons theory with complex gauge group. Of particular interest is a Chern-Simons theory with gauge group $G_{\mathbb{C}}=S L(2, \mathbb{C})$ (viewed as a complexification of $G=S U(2)$ ), which has many interesting connections with three-dimensional quantum gravity and geometry of hyperbolic three-manifolds. In this introductory section we review some aspects of these relations, formulate the problem, and describe various applications.

\subsection{Chern-Simons Theory}

Consider an oriented three-dimensional space $M$. We wish to formulate a ChernSimons gauge theory on $M$ with complex gauge group $G_{\mathbb{C}}$, whose real form we denote by $G$. Let $\mathbf{g}_{\mathrm{C}}$ and $\mathbf{g}$ be the corresponding Lie algebras. In these notations, the gauge connection $\mathcal{A}$ is a one-form on $M$ valued in the complex Lie algebra $\mathbf{g}_{\mathrm{C}}$. Explicitly, we can write $\mathcal{A}=\sum_{a} \mathcal{A}^{a} \cdot T_{a}$ where $T_{a}$ denote the generators of $\mathbf{g}$, which are assumed to be orthonormal, $\operatorname{Tr}\left(T_{a} T_{b}\right)=\delta_{a b}$. Then, the Chern-Simons action can be written as a sum of the holomorphic and anti-holomorphic terms,

$$
\begin{aligned}
I= & \frac{t}{8 \pi} \int_{M} \operatorname{Tr}\left(\mathcal{A} \wedge d \mathcal{A}+\frac{2}{3} \mathcal{A} \wedge \mathcal{A} \wedge \mathcal{A}\right)+ \\
& +\frac{\bar{t}}{8 \pi} \int_{M} \operatorname{Tr}\left(\overline{\mathcal{A}} \wedge d \overline{\mathcal{A}}+\frac{2}{3} \overline{\mathcal{A}} \wedge \overline{\mathcal{A}} \wedge \overline{\mathcal{A}}\right)
\end{aligned}
$$

where $t=k+i s$ and $\bar{t}=k-i s$ are the corresponding coupling constants. Consistency of the quantum theory requires the "level" $k$ to be an integer, $k \in \mathbb{Z}$. The other parameter, $s$, is not quantized. However, $s$ must obey certain constraints imposed by unitarity [1].

In Euclidean space, unitarity implies that the argument of the Feynman path integral

$$
Z(M)=\int \mathcal{D} \mathcal{A} e^{i I}
$$

must be complex conjugated under a reversal of the orientation on $M$. In the Chern-Simons theory defined by the action (1.1), there are two possibilities to achieve this, corresponding to either purely real or purely imaginary values of $s$. In the first case, $\mathcal{A}$ is invariant under the reversal of the orientation and $\bar{t}$ is the usual complex conjugate of $t$. On the other hand, the second possibility, $s \in i \mathbb{R}$, is realized when the gauge connection transforms nontrivially under the reversal of the orientation, $\mathcal{A} \mapsto \overline{\mathcal{A}}$. In the rest of the paper we mainly consider the case of imaginary $s$ and $G_{\mathbb{C}}=S L(2, \mathbb{C})$, which is related to the Euclidean quantum gravity in three dimensions (see discussion below). 


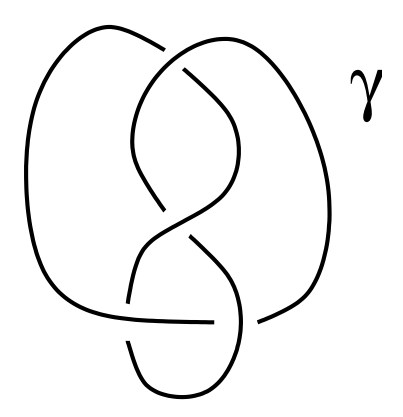

Fig. 1: A knotted Wilson loop in the $\mathbb{R}^{3}$.

Now let us consider observables in this theory. If $M$ is a manifold without boundary, holonomies of the gauge connection provide a complete set of observables, also known as "Wilson lines" in the context of gauge theory or "loop variables" in the context of gravity [2]. Specifically, given a closed oriented curve $\gamma \subset M$ and a representation $R$ of $G_{\mathbb{C}}$, one can define a gauge invariant observable as

$$
W_{R}(\gamma)=\operatorname{Tr}_{R} \operatorname{Hol}_{\gamma}(\mathcal{A})=\operatorname{Tr}_{R}\left(P \exp \oint_{\gamma} \mathcal{A}\right)
$$

The Wilson loop observables $W_{R}(\gamma)$ are naturally associated with knots in $M$. Indeed, even though intrinsically the curve $\gamma$ is simply a circle, its embedding in $M$ may be highly non-trivial, represented by a knot, as in fig. 1. More generally, an embedding of a collection of circles into $M$ is called a link, and the image of each circle is called a component of the link. Thus, given a link with (non-intersecting) components $\gamma_{i}, i=1, \ldots, r$, and a set of representations $R_{i}$ assigned to each component of the link, one can study a natural generalization of the Feynman path integral (1.2):

$$
Z\left(M ; \gamma_{i}, R_{i}\right)=\int \mathcal{D} \mathcal{A} \exp (i I) \prod_{i=1}^{r} W_{R_{i}}\left(\gamma_{i}\right)
$$

which, following [3], we call the (unnormalized) expectation value of the link. By construction, it is a function of $t$ and $\bar{t}$, which also depends on the topology of the three-manifold $M$, on the choice of the Wilson lines $\gamma_{i}$, and on the corresponding representations $R_{i}$.

A large class of representations $R_{i}$ can be naturally obtained by complexification from the corresponding representations of the real Lie algebra $\mathbf{g}$. However, no new information can be gained by studying such representations since the evaluation of (1.4) essentially reduces to the Chern-Simons theory with the real form of the gauge group, $G$, at least 
in perturbation theory 1 . On the other hand, of particular interest are certain infinite dimensional representations $R_{i}$ that we describe explicitly in the next subsection, after explaining the connection with three-dimensional quantum gravity.

\subsection{Three-Dimensional Quantum Gravity}

As we already mentioned earlier, there are several intriguing connections between three-dimensional gravity and Chern-Simons theory with complex gauge group $G_{\mathbb{C}}=$ $S L(2, \mathbb{C})$ and imaginary values of the parameter $s$ (for convenience, in what follows we shall use a real parameter $\sigma=i s)$. Thus, $S L(2, \mathbb{C})$ appears as the Poincare group in three-dimensional Euclidean gravity with negative cosmological constant 2 . Moreover, by writing the complex gauge field $\mathcal{A}$ in terms of the real and imaginary components one can relate the Chern-Simons action (1.1) to the usual form of the Einstein-Hilbert action of three-dimensional gravity with negative cosmological constant [6,7]. Specifically, writing $\mathcal{A}=w+i e$ and $\overline{\mathcal{A}}=w-i e$ one finds

$$
\begin{aligned}
I= & \frac{k}{4 \pi} \int_{M} \operatorname{Tr}\left(w \wedge d w-e \wedge d e+\frac{2}{3} w \wedge w \wedge w-2 w \wedge e \wedge e\right)+ \\
& +\frac{i \sigma}{2 \pi} \int_{M} \operatorname{Tr}\left(w \wedge d e+w \wedge w \wedge e-\frac{1}{3} e \wedge e \wedge e\right)
\end{aligned}
$$

The second term in this expression is indeed equivalent to the Einstein-Hilbert action 3 with negative cosmological constant, $\Lambda=-1$, written in terms of the vielbein $e$ and the spin connection $w$. We can also write it in the standard form:

$$
I_{\text {grav }}=-\frac{1}{4 \pi} \int_{M} d^{3} x \sqrt{g}(R+2)
$$

As will be shown below, the first term in (1.5) also has a nice interpretation. It is related to the Chern-Simons invariant of the three-manifold $M$. Therefore, it is convenient to denote this term as $I_{\mathrm{CS}}$. In the new notations, we can write the original action (1.1) as

$$
I(k, \sigma)=k I_{\mathrm{CS}}+i \sigma I_{\mathrm{grav}}
$$

1 See e.g. exercise 6.32 in [4].

2 More precisely, $\operatorname{Isom}_{+}\left(\mathbb{H}^{3}\right)=\operatorname{PSL}(2, \mathbb{C})=S L(2, \mathbb{C}) /\{ \pm 1\}$, but according to [5], a representation of $\operatorname{PSL}(2, \mathbb{C})$ corresponding to a complete hyperbolic structure can always be lifted to $S L(2, \mathbb{C})$, and it is $S L(2, \mathbb{C})$ with which we shall work.

3 In our notations, the length scale $\ell=1$ and the Newton constant $G_{N}=1 /(4 \sigma)$. 
Summarizing, following Witten [7], we conclude that the real and imaginary components of the $S L(2, \mathbb{C})$ Chern-Simons action (1.1) have a nice physical interpretation. In particular, a theory with $k=0$ represents, at least (semi-)classically, a three-dimensional Euclidean quantum gravity with negative cosmological constant $\$$. However, this equivalence does not readily extend to quantum theories due to a number of subtle issues, typically related to degenerate vielbeins etc. (see e.g. [10] for a recent discussion of these questions). For example, in the Chern-Simons theory, it is natural to expand around a trivial vacuum, $\mathcal{A}=0$, which corresponds to a very degenerate metric, $g_{i j}=0$. Also, in the Chern-Simons path integral (1.2) one integrates over all (equivalence classes of) gauge connections, whereas in quantum gravity one takes only a subset of those corresponding to positive-definite volume elements. Nevertheless, one would hope that, for certain questions, the relation to gravity can still be helpful even beyond the classical limit. Thus, in order to avoid the above problems throughout the paper we shall mainly consider the semi-classical expansion around an isolated critical point, corresponding to a non-degenerate metric on $M$. Then, the quantum fluctuations are small, and both theories are expected to agree.

So far we discussed a relation between $S L(2, \mathbb{C})$ Chern-Simons theory and pure gravity. Now let us add sources representing point particles. Assuming that particles don't have any internal structure, they can be characterized by two numbers: a mass and a spin. As we will see later, it is natural to combine these numbers into a single complex quantity, which labels an infinite dimensional representation of $S L(2, \mathbb{C})$, see [11, 12]. Interacting with gravity, matter particles produce conical defects in the geometry of the space manifold $M$ [13, 14, 15. In particular, light-like particles correspond to cusps in $M$. We shall say more about this aspect later, when we will be talking about the relation to hyperbolic geometry.

The coupling of point-like sources to gravity can be described by Wilson lines in the Feynman path integral (1.4), see e.g. [16, 17, 18, 19]. For example, if we introduce extra variables $p_{a}$ and $x^{a}$, which represent momentum and coordinate of a particle in space $M$, then the Wilson line operator for a spinless particle can be explicitly written as [16]:

$$
W_{R}(\gamma)=\int \mathcal{D} x^{a}(s) \mathcal{D} p_{a}(s) \mathcal{D} \lambda(s) \exp \left(i I_{W}\right)
$$

4 Chern-Simons theory with $S L(2, \mathbb{C})$ gauge group and real values of the parameter $s$ is also related to three-dimensional gravity, namely to de Sitter gravity in $2+1$ dimensions [6, []. This theory can be treated similarly, and many of the arguments below easily extend to this case. For work on quantization of this theory see [1, 8, 9]. 
where $s$ is a parameter along the "world-line" $\gamma$, and $I_{W}$ is the action

$$
I_{W}=\int_{\gamma} d s\left(p_{a} \frac{D x^{a}}{D s}-\lambda\left(\vec{p}^{2}-m^{2}\right)\right)
$$

In this action, $\lambda$ is a Lagrange multiplier that enforces the on-shell condition $\vec{p}^{2}=m^{2}$, and the coupling to gravity is encoded in the covariant derivative

$$
\frac{D x^{a}}{D s}=\frac{d x^{a}}{d s}+w_{s}^{a}{ }_{b} x^{b}+i e_{s}^{a}
$$

where $\left(e_{s}, w_{s}\right)$ denotes the restriction of $(e, w)$ to $\gamma$.

Eqs. (1.8) - (1.9) define an infinite dimensional representation $R$ of $S L(2, \mathbb{C})$ corresponding to a spinless particle in three dimensions. In a similar way, one can define a general class of infinite dimensional representations corresponding to massive spinning particles [18] and black holes, which is precisely the class of representations we are going to assign to Wilson lines $W_{R_{i}}\left(\gamma_{i}\right)$ in the Feynman path integral (1.4). Below we shall give an equivalent geometric description of such system.

\subsection{The Hartle-Hawking Wave Function in $2+1$ Dimensions}

There is yet another relation between $S L(2, \mathbb{C})$ Chern-Simons theory with $t=-\bar{t}=\sigma$ and three dimensions gravity with negative cosmological constant. Namely, in the HartleHawking no-boundary proposal [20], the ground state wave function of the $2+1$ dimensional Lorentzian universe is obtained from the Euclidean path integral over metrics on a 3manifold $M$ with boundary $\Sigma=\partial M$,

$$
\Psi_{\Sigma}(h)=\sum_{M} \int \mathcal{D} g \exp \left(-\sigma I_{\text {grav }}\right)
$$

Here, the summation represents a sum over topologies, and $I_{\text {grav }}$ is the Euclidean gravity action (1.6). Again, we consider pure gravity without coupling to matter. The functional $\Psi_{\Sigma}(h)$, called the Hartle-Hawking wave function, is a functional of the induced metric $h$ on the space-like surface $\Sigma$. It should be interpreted as an amplitude of finding the universe in a quantum state, characterized by the spatial geometry $\Sigma$ with metric $h$, as schematically shown on the figure below. Furthermore, a "smooth" continuation from the Riemannian to Lorentzian metrics across $\Sigma$ is possible only if we limit the sum (1.10) to manifolds $M$ such that the boundary $\Sigma=\partial M$ is totally geodesic [21]. 


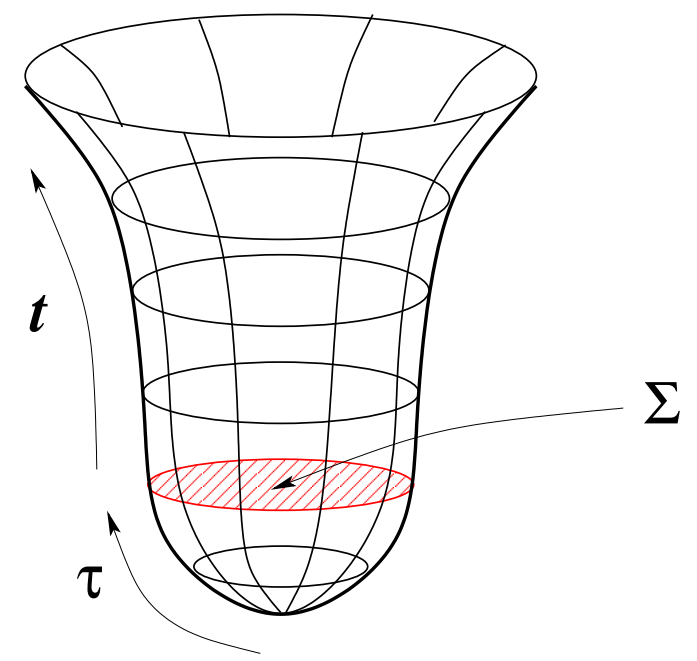

Fig. 2: In the Hartle-Hawking no-boundary proposal, the "analytic continuation" across a surface $\Sigma$ describes a real tunneling from "nothing" to a universe with "space" $\Sigma$.

The wave function (1.10) obeys the Wheeler-DeWitt equation [22], which has the form of the Hamiltonian constraint

$$
\widehat{H} \Psi=0
$$

where $\widehat{H}$ is a second-order differential operator determined by the topology of the surface $\Sigma$. For instance, when $\Sigma$ is a torus, $\widehat{H}$ essentially reduces to the Laplace operator on the torus moduli space [23], see also [24,25, 26, 27,28].

The case when $\Sigma=T^{2}$ (or a collection of tori) turns out to be closely related to the problem of computing the Chern-Simons path integral (1.4) with Wilson loops. Indeed, modulo the subtleties related to the integration domain in (1.10), one could replace the integral over the metrics by the Chern-Simons path integral of the form (1.2). This would give a wave functional

$$
\Psi_{\Sigma}(h)=\sum_{M} Z(M ; \Sigma)
$$

where each term $Z(M ; \Sigma)$ depends on the topology of the three-manifold $M$ and on the boundary conditions at $\Sigma=T^{2}$. These boundary data can be identified with the parameters of the Wilson line $W_{R}(\gamma)$ in the infinite dimensional representation $R$ of $S L(2, \mathbb{C})$, so that one has [29]:

$$
Z\left(M ; T^{2}\right)=Z\left(\mathbf{S}^{3} ; \gamma, R\right)
$$

where $M=\mathbf{S}^{3} \backslash \gamma$ is a compact three-manifold with a single torus boundary, obtained by removing a Wilson line from the 3-sphere. Indeed, one can split the path integral 
on the right-hand side of (1.13) into three parts corresponding to: 1) the integral over the connection $\mathcal{A}$ inside a small neighborhood of the Wilson line; 2) the integral over its complement, $M=\mathbf{S}^{3} \backslash \gamma$, with certain boundary conditions on the boundary, $\partial M=T^{2}$; 3 ) and, finally, the integral over these boundary conditions. Then, the first integral leads to a delta-function that fixes the boundary conditions to certain values, so that the entire path integral can be reduced to $Z\left(M ; T^{2}\right)$ with certain boundary conditions on the $T^{2}$.

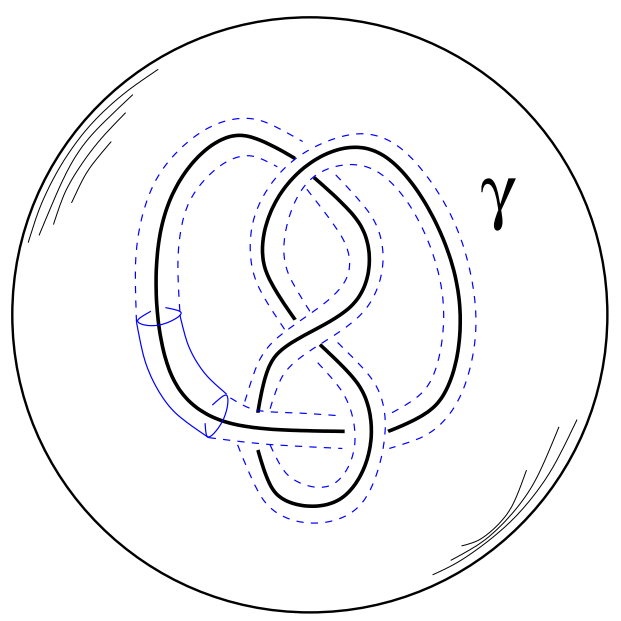

Fig. 3: A compact 3-manifold $M$ with a single torus boundary can be constructed by removing a small neighborhood of a knotted Wilson loop from the 3-sphere.

It is natural to expect that the wave function (1.12) obtained from the Chern-Simons path integral satisfies an equation of the form (1.11). A more optimistic hope is that each term, $Z(M ; \Sigma)$, individually solves an analog of the Wheeler-DeWitt equation. As we shall see below, this is indeed the case, and the generalized partition function (11.4) indeed obeys a functional equation of the form (1.11). Finally, we note that, even though in this work we mainly focus on knot complements, the arguments can be extended to generic 3 -manifolds with arbitrary boundary $\Sigma$ (in particular, to 3-manifolds with infinite volume). These manifolds also have a nice interpretation in three-dimensional gravity [30], and will be briefly discussed in section 4 and in Appendix A.

\subsection{Quantum Geometry of Hyperbolic Three-Manifolds}

Note, that the definition of the path integral (1.4) does not involve a choice of metric on $M$. Therefore, $Z\left(M ; \gamma_{i}, R_{i}\right)$ is expected to be a topological invariant of the three-manifold $M$ with a link, whose components $\gamma_{i}$ are colored by (infinite-dimensional) representations, $R_{i}$, of the complex gauge group $G_{\mathbb{C}}$. From the physical point of view, this is not very 
surprising since we start with a Chern-Simons theory, which has vanishing Hamiltonian and, therefore, is expected to be a topological quantum field theory.

The quantum invariant $Z\left(M ; \gamma_{i}, R_{i}\right)$ is particularly interesting for the complex gauge group $G_{\mathbb{C}}=S L(2, \mathbb{C})$, which is related to three-dimensional Euclidean gravity with negative cosmological constant. In particular, classical solutions in the gravity theory (1.6) correspond to manifolds with constant negative curvature, and one can also interpret certain Wilson line operators $W_{R}(\gamma)$ as operators creating cusps in $M$. Therefore, $Z\left(M ; \gamma_{i}, R_{i}\right)$ is a natural invariant of hyperbolic three-manifolds with cusps. If we remove the cusps from $M$, we can also interpret $Z\left(M ; \gamma_{i}, R_{i}\right)$ as a topological invariant of the complement of the link, whose components are $\gamma_{i}$.

According to a famous theorem by Thurston, many 3-manifolds are hyperbolic [31]. For example, the complement of a knot in $\mathbf{S}^{3}$ admits a hyperbolic structure unless it is a torus or satellite knot. Moreover, after the Mostow Rigidity Theorem [32], any geometric invariant of a hyperbolic 3-manifold is a topological invariant. Important geometric invariants, which can be defined even if the hyperbolic manifold $M$ has cusps [33], are the volume and the Chern-Simons invariant. Thurston suggests to combine these two invariants into a single complex invariant $Z(M)$ whose absolute value is $e^{2 / \pi \operatorname{Vol}(M)}$ and whose argument is the Chern-Simons invariant of $M$ [31]:

$$
Z(M) \sim \exp \left(\frac{2}{\pi} \operatorname{Vol}(M)+4 \pi i C S(M)\right)
$$

As one might expect from (1.7), the path integral (1.2) reduces to an invariant like this in the semi-classical limit.

More surprisingly, extending the well-known volume conjecture 34, 35, 36, we find that the $N$-colored Jones polynomial, $J_{N}\left(\gamma, e^{2 \pi i / k}\right)$, also has a similar asymptotic behavior in the limit $k, N \rightarrow \infty$, such that the ratio, $a=N / k$, is fixed. Namely, depending on whether the parameter $a$ is rational or not, the asymptotic behavior of $J_{N}\left(e^{2 \pi i / k}\right)$ is either polynomial or exponential (the value $a=1$ is somewhat special). According to a seminal work of Witten [3], the first case is related to the $S U(2)$ Chern-Simons gauge theory and rational conformal field theory in two dimensions. On the other hand, as we show below, the exponential growth of the colored Jones polynomial encodes a lot of interesting information about the hyperbolic geometry of the knot complement and, therefore, is more suggestive of the $S L(2, \mathbb{C})$ Chern-Simons theory. Schematically, the whole picture can be summarized in the following table: 


\begin{tabular}{|c|c|c|c|}
\hline$a=N / k$ & $\begin{array}{c}\text { asymptotic behavior } \\
\text { of } J_{N}\left(e^{2 \pi i / k}\right)\end{array}$ & CFT & $\begin{array}{c}\text { Chern-Simons } \\
\text { theory }\end{array}$ \\
\hline Rational & Polynomial & Rational & $S U(2)$ \\
\hline Non-Rational & Exponential & Non-Rational & $S L(2, \mathbb{C})$ \\
\hline
\end{tabular}

Table 1: Asymptotic behavior of the colored Jones polynomial for hyperbolic knots.

This heuristic picture agrees with the fact that, when $k=0$ and $\sigma \in \mathbb{Z}$, the partition function (1.2) of the $S L(2, \mathbb{C})$ Chern-Simons theory can be formally regarded as a product of two $S U(2)$ partition functions by treating $\mathcal{A}$ and $\overline{\mathcal{A}}$ as independent $S U(2)$ gauge fields. Moreover, it was argued in [37] that at these values of the coupling constants the Hilbert space of the $S L(2, \mathbb{C})$ Chern-Simons theory on a torus factorizes into two copies of the Hilbert space of an $S U(2)$ theory.

The analytic continuation to non-integer values of $k$ in the $S U(2)$ Chern-Simons theory can be also motivated by connection with topological string theory. Just like ordinary quantum field theory can be embedded in string theory, topological Chern-Simons theory can be realized in topological open string theory [38], where the string coupling constant is related to the level $k$. In many cases, these theories admit a dual description in terms of closed topological strings on a (non-compact) Calabi-Yau manifold [39]. Therefore, from the point of view of topological string theory, it is also natural (and sometimes even necessary) to consider non-integer values of $k$, which via duality is identified with the complexified Kähler parameter of the Calabi-Yau manifold [40]. Then, the results of this paper suggest that certain invariants of hyperbolic 3-manifolds might emerge from topological closed string theory in the "zero radius limit".

Finally, we note that Chern-Simons theory with $S L(2, \mathbb{C})$ gauge group also has a number of interesting applications in string theory, see e.g. [41,42] for some recent work.

\section{Organization of the Paper}

The rest of the paper is organized as follows: In section 2, we study classical aspects of Chern-Simons theory with complex gauge group. In particular, we introduce the Apolynomial of a knot and identify it with the space of classical solutions in the $S L(2, \mathbb{C})$ Chern-Simons theory on the knot complement. Section 3 is devoted to quantization of this theory (in a real polarization). Starting with section 4, we discuss various extensions 
and applications of this approach, including mathematical applications. In section 5, we present some evidence for the picture summarized in Table 1 and propose a generalization of the volume conjecture to incomplete hyperbolic structures on knot complements. Further aspects of the relation with $S U(2)$ Chern-Simons theory and the colored Jones polynomial are discussed in section 6, where we propose an analog of the Melvin-Morton-Rozansky conjecture for a non-trivial ("hyperbolic") flat connection. Finally, in the appendices we illustrate the general ideas using simple examples. Thus, in Appendix A, we use the geometry of the BTZ black hole to explain the origin and the interpretation of the Apolynomial for the trivial knot. In Appendix B, we discuss quantization of the $S L(2, \mathbb{C})$ Chern-Simons theory for torus knots, which leads to a particularly simple (Gaussian) quantum mechanics.

\section{Classical Theory}

We wish to study quantum Chern-Simons theory (1.1) with Wilson lines in the infinite dimensional representations of the complex gauge group $G_{\mathbb{C}}$. Like in any constrained system, there are two ways of quantizing the theory: one can either quantize the classical phase space (that is the space of solutions of the classical equations of motion); or one can impose the constraints after quantization. See e.g. 43 for a nice exposition of both methods. Here, we will follow the first approach. For this, we need to begin by studying the classical solutions of the theory; this will be the first place where we encounter the A-polynomial.

\subsection{The Moduli Space of Classical Solutions}

Away from the location of Wilson loops, the classical Euler-Lagrange equations derived from the Chern-Simons action (1.1) look like

$$
\mathcal{F}=\overline{\mathcal{F}}=0
$$

where $\mathcal{F}=d \mathcal{A}+\mathcal{A} \wedge \mathcal{A}$ is the field strength, and $\overline{\mathcal{F}}$ is its complex conjugate. Therefore, the classical solutions in Chern-Simons theory are the so-called flat connections, i.e. the gauge fields with zero curvature.

A flat connection on $M$ is determined by its holonomies, that is by a homomorphism

$$
\rho: \pi_{1}(M) \rightarrow G_{\mathbb{C}}
$$


Hence, the moduli space of classical solutions is given by the set of representations of the fundamental group, $\pi_{1}(M)$, into the group $G_{\mathbb{C}}$ modulo gauge transformations, which act on $\rho$ by conjugation,

$$
L=\operatorname{Rep}\left(\pi_{1}(M) \rightarrow G_{\mathbb{C}}\right) / \text { conjugation }
$$

Similarly, if $M$ is a compact 3-manifold with boundary, $\partial M=\Sigma$, one can consider the moduli space of flat connections on $\Sigma$,

$$
\mathcal{P}=\operatorname{Rep}\left(\pi_{1}(\Sigma) \rightarrow G_{\mathbb{C}}\right) / \text { conjugation }
$$

There is a natural map from the representation variety $L$ to $\mathcal{P}$ induced by restricting a flat connection on $M$ to $\Sigma$. The image of $L$ under this map is a middle-dimensional submanifold in $\mathcal{P}$. In the canonical quantization, that will be discussed in the next section, $\mathcal{P}$ is a classical phase space, whereas the image of $L$ is associated with a semi-classical state.

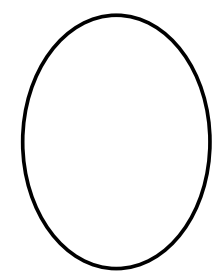

unknot

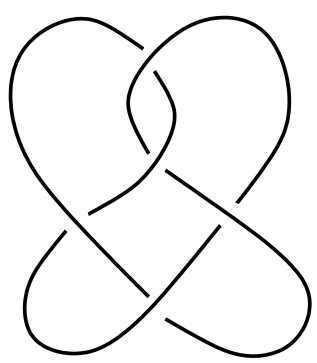

tweeny knot $\mathbf{5}_{2}$

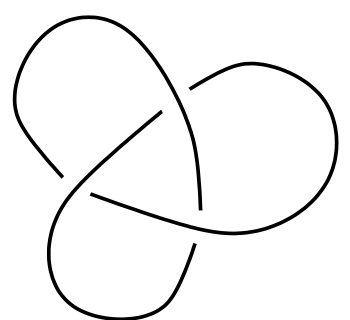

trefoil knot $3_{1}$

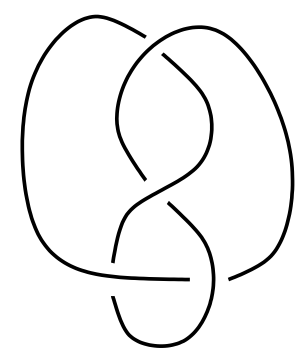

figure-8 knot $4_{1}$

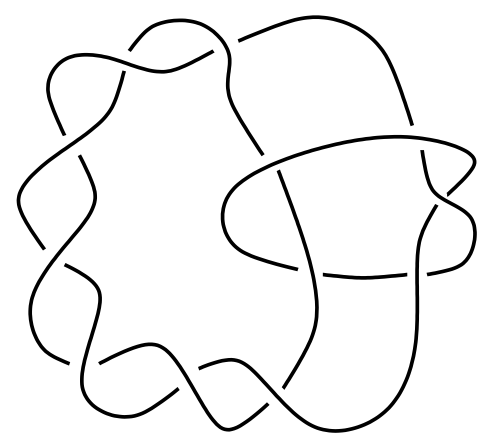

pretzel knot $(-2,3,7)$

Fig. 4: Some simple knots in three-dimensional space. We use the standard notation $\mathbf{k}_{n}$ to indicate the $n$-th knot in the census of knots with $k$ crossings. 
For simplicity, in what follows we focus on a large class of compact oriented 3-manifolds with a single torus boundary component, $\Sigma=T^{2}$. In particular, $M$ can be an exterior of a knotted Wilson loop, which we denote by $\gamma=K$, in a rational homology sphere. For example, see fig. 4 for some simple knots in the $\mathbf{S}^{3}$. Specifically, let $N(K)$ be a tubular neighborhood of the knot $K$; it is homeomorphic to a solid torus, $N \cong \mathbf{D}^{2} \times \mathbf{S}^{1}$. Then, the knot complement (also known as a knot exterior),

$$
M=\mathbf{S}^{3} \backslash N(K)
$$

is a compact 3-manifold whose boundary is a torus, $\Sigma=T^{2}$. (This construction reminds the creation of the so-called "stretched horizon" in the context of Euclidean gravity [44,45].) The group $\pi_{1}(M)$ is usually called the knot group, and $\pi_{1}(\Sigma)$ is called the peripheral subgroup of $M$.

\section{2. $S L(2, \mathbb{C})$ Chern-Simons Theory and the A-polynomial}

Of particular interest is a Chern-Simons theory with complex gauge group $G_{\mathbb{C}}=$ $S L(2, \mathbb{C})$ due to its relation to three-dimensional Euclidean gravity. Indeed, as we already mentioned in the previous section, the $S L(2, \mathbb{C})$ Chern-Simons action (1.1) with $t=-\bar{t}=\sigma$ is equivalent to the Einstein-Hilbert action, $I_{\text {grav }}$, written in the first order formalism. Correspondingly, the classical field equations (2.1) in the Chern-Simons theory have the form of the usual Einstein equations in three-dimensional general relativity with negative cosmological constant, $\Lambda=-1$,

$$
R_{i j}=-2 g_{i j}
$$

written in the first-order formalism. In three dimensions, the full curvature tensor is completely determined by the Ricci tensor 5 and (2.6) implies that $g_{i j}$ is a metric with constant negative curvature.

Therefore, hyperbolic metrics on a three-manifold $M$ can be naturally viewed as classical solutions to the field equations (2.1) in the Chern-Simons theory. Any hyperbolic manifold is locally isometric to the hyperbolic three-space, $\mathbb{H}^{3}$. We remind that $\mathbb{H}^{3}$ can be defined as the upper half-space with the standard hyperbolic metric

$$
d s^{2}=\frac{1}{z^{2}}\left(d x^{2}+d y^{2}+d z^{2}\right) \quad, \quad z>0
$$

5 One has the following identity: $R_{i j k l}=g_{i k} R_{j l}+g_{j l} R_{i k}-g_{j k} R_{i l}-g_{i l} R_{j k}-\frac{1}{2}\left(g_{i k} g_{j l}-g_{i l} g_{j k}\right) R$. 
If a hyperbolic space $M$ is geodesically complete, it can be represented as a quotient space,

$$
M=\mathbb{H}^{3} / \Gamma
$$

where a discrete, torsion-free subgroup $\Gamma \subset P S L(2, \mathbb{C})$ is a holonomy representation of the fundamental group $\pi_{1}(M)$ into $\operatorname{Isom}_{+}\left(\mathbb{H}^{3}\right)=P S L(2, \mathbb{C})$. Fortunately, every holonomy representation lifts to a representation (2.2) into the matrix group $S L(2, \mathbb{C})$, which is much easier to deal with [5,31]. In particular, the action of $\Gamma$ on $\mathbb{H}^{3}$ can be conveniently expressed by identifying a point $(x, y, z) \in \mathbb{H}^{3}$ with a quaternion $q=x+y \mathbf{i}+z \mathbf{j}$ and writing

$$
q \mapsto(a q+b) /(c q+d) \quad, \quad\left(\begin{array}{ll}
a & b \\
c & d
\end{array}\right) \in S L(2, \mathbb{C})
$$

Since our main examples are three-manifolds (2.5) with a single torus boundary, which can be represented as knot complements in a three-sphere, it is natural to ask if such manifolds can admit a hyperbolic structure. It turns out that many of them can. Namely, a famous theorem of Thurston says that, unless $K$ is a torus or a satellite knot, its complement admits a hyperbolic metric [31]. Such knots are called hyperbolic.

\section{Example. The Figure-eight Knot}

Among all hyperbolic knots, a complement of the figure-eight knot $4_{1}$ (see fig. 4) has the least possible volume: $\operatorname{Vol}\left(4_{1}\right)=2.0298832128 \ldots$, and admits a decomposition into two regular ideal tetrahedra, $M=\Delta_{0} \cup \Delta_{0}$. The knot group, $\pi_{1}(M)$, is generated by two elements, $a$ and $b$, such that $a^{-1} b a b^{-1} a b=b a^{-1} b a$. The corresponding representation into $S L(2, \mathbb{C})$ is given by

$$
\rho(a)=\left(\begin{array}{ll}
1 & 1 \\
0 & 1
\end{array}\right) \quad \text { and } \quad \rho(b)=\left(\begin{array}{cc}
1 & 0 \\
\frac{1-\sqrt{-3}}{2} & 1
\end{array}\right)
$$

The complement of the figure-eight knot can be also represented as a quotient space (2.8), where the holonomy group $\Gamma$ is generated by the above two matrices.

Therefore, a complete hyperbolic structure on the complement (2.5) of a hyperbolic knot $K \subset \mathbf{S}^{3}$ can be naturally associated with a flat connection in $S L(2, \mathbb{C})$ Chern-Simons theory with a Wilson loop $K$ or, equivalently, in a theory without the Wilson loop, defined on its complement, $M=\mathbf{S}^{3} \backslash K$. However, it is important to stress here that $S L(2, \mathbb{C})$ Chern-Simons theory - which is the main subject of this paper - makes sense even if the knot $K$ is not hyperbolic. In particular, in Appendix B we will discuss torus knots. Even though such examples are not related to hyperbolic geometry, one can still study flat $S L(2, \mathbb{C})$ connections and their moduli spaces, (2.3) and (2.4). This is what we are going to do next for an arbitrary knot $K$. 


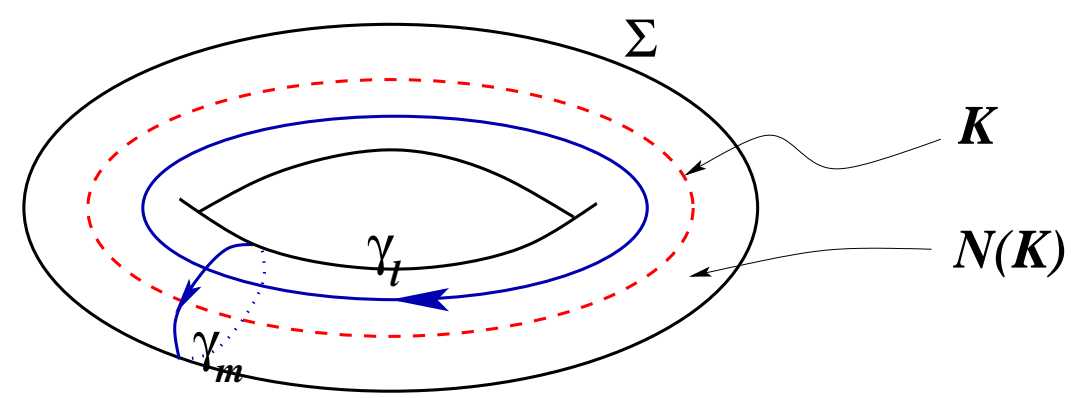

Fig. 5: The longitude $\gamma_{l}$ and the meridian $\gamma_{m}$ furnish a basis of the peripheral subgroup $\pi_{1}(\Sigma)=\mathbb{Z} \times \mathbb{Z}$.

In the case of $S L(2, \mathbb{C})$ Chern-Simons theory defined on a knot complement $M$ we can give a rather explicit description of the representation variety, $L$, and the classical phase space, $\mathcal{P}$. Since the boundary of $M$ is a torus, $\Sigma=T^{2}$, there are two simple closed curves, $\gamma_{l}$ and $\gamma_{m}$, on $M$ called the longitude and meridian, which intersect transversely in a single point, see fig. 5. These two curves generate the peripheral subgroup of $M$, which is a free abelian group $\pi_{1}(\Sigma)=\mathbb{Z} \times \mathbb{Z}$. Therefore, a representation, $\rho$, of this group into $S L(2, \mathbb{C})$ can be conjugated to the upper triangular matrices

$$
\rho\left(\gamma_{l}\right)=\left(\begin{array}{cc}
l & * \\
0 & 1 / l
\end{array}\right) \quad, \quad \rho\left(\gamma_{m}\right)=\left(\begin{array}{cc}
m & * \\
0 & 1 / m
\end{array}\right)
$$

where $l$ and $m$ are complex numbers. No further conjugacy of $\rho$ is possible 6 , so that we can say that the pair of 'eigenvalues' $(l, m)$ is the invariant data parametrizing the representation of the peripheral subgroup into $S L(2, \mathbb{C})$. This also gives an implicit description of the infinite dimensional representations that we associate with Wilson loops $W_{R}(K)$ in the Chern-Simons path integral (1.4). In order to emphasize the explicit dependence on $l$ and $m$, in what follows we sometimes refer to such Wilson loops as $W_{(l, m)}(K)$. Depending on the values of the holonomies, such Wilson lines can be interpreted as either black holes or massive particlest in three-dimensional gravity. On the other hand, in the hyperbolic geometry of the 3-manifold $M$, such Wilson lines correspond to torus boundaries (cusps). Roughly, the complex numbers $l$ and $m$ parameterize the "length" and the "torsion" of the cusp.

\footnotetext{
6 Following [46], in our discussion we suppress the quotient by the Weyl group.

7 Namely, a Wilson line should be interpreted as a massive particle when the holonomy $\rho\left(\gamma_{m}\right)$ is elliptic, and as a spinning black hole otherwise, see e.g. 447. I wish to thank K. Krasnov for useful discussions on various points related to this interpretation.
} 
Therefore, we find that for a compact oriented 3-manifold $M$ with a single torus boundary the classical phase space $\mathcal{P}$ is a two-dimensional complex manifold

$$
\mathcal{P}=\mathbb{C}^{*} \times \mathbb{C}^{*}
$$

parametrized by the eigenvalues $(l, m)$ of the holonomies (2.10). Notice, that the phase space $\mathcal{P}=\mathbb{C}^{*} \times \mathbb{C}^{*}$ can be regarded as the total space of the cotangent bundle,

$$
\mathcal{P}=T^{*}\left(\mathbf{S}^{1} \times \mathbf{S}^{1}\right)
$$

where the two circles are parametrized by $\arg (l)$ and $\arg (m)$, and the directions along the fiber are parametrized by $\log |l|$ and $\log |m|$. Here, the base manifold,

$$
\mathbf{S}^{1} \times \mathbf{S}^{1}=\operatorname{Hom}\left(\pi_{1}(\Sigma) ; S U(2)\right) / S U(2)
$$

can be viewed as a representation space of the peripheral subgroup, $\pi_{1}(\Sigma)$, into the compact part of the gauge group, $G=S U(2)$.

Our next task is to describe the representation variety,

$$
L=\operatorname{Hom}\left(\pi_{1}(M) ; S L(2, \mathbb{C})\right) / S L(2, \mathbb{C})
$$

Thurston showed that for a space $M$ with a single torus boundary, the dimension of the numerator in this formula is equal to 4 . On the other hand, the Lie group $S L(2, \mathbb{C})$ has complex dimension 3. Therefore, after we identify conjugate representations in (2.14) we obtain a variety of complex dimension one. Furthermore, a basis $\left(\gamma_{l}, \gamma_{m}\right)$ for the peripheral subgroup of $M$ determines an embedding of $L$ into $\mathcal{P}=\mathbb{C}^{*} \times \mathbb{C}^{*}$, and using the standard techniques from algebraic geometry one can show that the variety $L$ is the zero locus of a single polynomial $A(l, m)$ in two variables, the so-called A-polynomial [46]. In a sense, in the $S L(2, \mathbb{C})$ Chern-Simons theory the A-polynomial plays a role similar to the role of the Seiberg-Witten curve in $\mathcal{N}=2$ supersymmetric gauge theory [48], which also describes a moduli space of vacua. In fact, as we shall see below, interesting physical quantities in the $S L(2, \mathbb{C})$ Chern-Simons theory can be also expressed in terms of the period integrals on the curve $L$ defined as the zero locus of the A-polynomial.

Explicitly, we can write $L$ as:

$$
L=\left\{(l, m) \in \mathbb{C}^{*} \times \mathbb{C}^{*} \mid A(l, m)=0\right\}
$$


In three-dimensional Euclidean gravity, the A-polynomial can be naturally interpreted as a mass shell condition for a "self-gravitating" massive spinning particle or a black hole propagating along a knot $K$ in the $\mathbb{R}^{3}$ (or in the 3 -sphere). For example, the A-polynomial of the unknot (trivial knot) is

$$
A_{\text {unknot }}(l, m)=l-1
$$

due to the abelian representations. Essentially, this result appears in the study of the off-shell BTZ black hole [49,50,51,28]. In Appendix A, we review the geometry of the BTZ black hole and reproduce the A-polynomial (2.16).

Since $H_{1}(M) \cong \mathbb{Z}$ for any knot complement (2.5), there is always a component of the character variety $L$ corresponding to abelian representations. Usually, one ignores this component, which contributes a factor of $(l-1)$ to the A-polynomial 8 . There is still some ambiguity in the definition of the A-polynomial. Namely, the A-polynomial is defined up to scaling and up to multiplication by powers of $l$ and $m$. It turns out, however, that there is a natural normalization, such that $A(l, m)$ is a polynomial with integer coefficients [46]. This is the normalization that will be used throughout this paper.

The explicit form of the A-polynomial depends on the knot $K$, and it can be always systematically computed [46]. A-polynomials of some simple knots are listed below:

\begin{tabular}{|c|c|c|}
\hline Knot & A-polynomial & Volume \\
\hline $3_{1}$ & $l m^{6}+1$ & non-hyperbolic \\
$4_{1}$ & $-2+m^{4}+m^{-4}-m^{2}-m^{-2}-l-l^{-1}$ & $2.0298832 \ldots$ \\
$5_{1}$ & $l m^{10}+1$ & non-hyperbolic \\
$5_{2}$ & $1+l\left(-1+2 m^{2}+2 m^{4}-m^{8}+m^{10}\right)+l^{2}\left(m^{4}-m^{6}+\right.$ & $2.8281220 \ldots$ \\
& $\left.+2 m^{10}+2 m^{12}-m^{14}\right)+l^{3} m^{14}$ & \\
$7_{1}$ & $m^{14}+l$ & non-hyperbolic \\
$(-2,3,7)$-pretzel & $-m^{110}+l m^{90}\left(m^{2}-1\right)^{2}+l^{2}\left(2 m^{74}+m^{72}\right)-$ & $2.8281221 \ldots$ \\
& $-l^{4}\left(m^{38}+2 m^{36}\right)-l^{5} m^{16}\left(m^{2}-1\right)^{2}+l^{6}$ & \\
\hline
\end{tabular}

Table 2: A-polynomials of some knots and the hyperbolic volumes of their complements.

8 However, in quantization of the theory it will be crucial to include all the components of $L$. I am grateful to G. Moore and D. Thurston for emphasizing this aspect. 
When the knot $K$ is hyperbolic we can interpret (a subset of) the zero locus of the A-polynomial as the moduli space of hyperbolic structures on the knot complement, $M=$ $\mathbf{S}^{3} \backslash K$. Most of the points in this moduli space correspond to incomplete hyperbolic metrics on $M$. However, there are some special values of $l$ and $m$, for which the metric on $M$ has extra nice properties. For instance, at the point $(l, m)=(-1,1)$ we obtain a complete hyperbolic manifold $M$, with a cusp along $K$. The volumes of such manifolds evaluated at this particular point in the moduli space are listed in Table 2.

Furthermore, if $l$ and $m$ satisfy an equation of the form

$$
l^{p} m^{q}=1
$$

for some co-prime integers $p$ and $q$, then the knot complement $M$ can be completed into a compact space $\bar{M}$ without boundary, which can be also obtained by performing $(p, q)$-Dehn surgery on the knot $K$ in the 3 -sphere:

$$
\bar{M}=M(p, q)
$$

Specifically, let $N(K)$ be the tubular neighborhood of the knot $K$, as on fig. 5, and let $\gamma_{l}$ and $\gamma_{m}$ be the longitude and the meridian of the boundary torus. Then, the $(p, q)$ Dehn surgery on $K$ is obtained by replacing $N(K)$ with another solid torus, such that the meridian in the solid torus matches up with the curve $\gamma_{l}^{p} \gamma_{m}^{q}$ in $\partial M$ :

$$
M(p, q)=\left(\mathbf{S}^{3} \backslash N(K)\right) \cup_{T^{2}} \mathbf{D}^{2} \times \mathbf{S}^{1}
$$

Except for a finite number of cases, the resulting 3-manifold $M(p, q)$ admits a hyperbolic metric. The volume of the space $M(p, q)$ is always smaller than the volume of the "parent" manifold $M_{\text {cusped }}$ with a cusp, and as $p^{2}+q^{2} \rightarrow \infty$ the volume of $M(p, q)$ approaches the volume of $M_{\text {cusped }}$, as illustrated on the figure below:

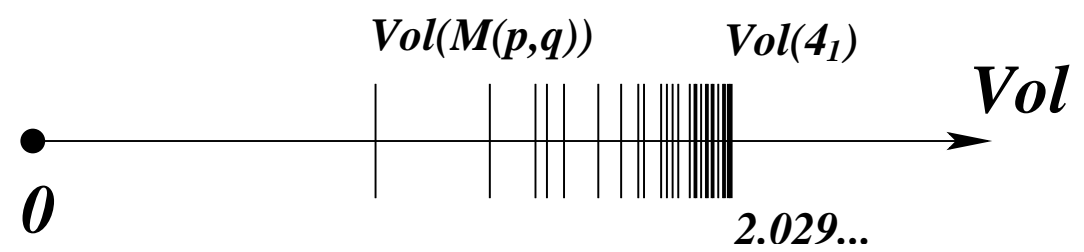

Fig. 6: The volumes of the hyperbolic 3-manifolds $M(p, q)$ obtained by Dehn surgery on the figure-eight knot converge to the volume of the cusped manifold. 
For example, for the figure-eight knot the sequence $\operatorname{Vol}(M(p, q))$ looks like [52]:

$$
\operatorname{Vol}(M(p, q))=\operatorname{Vol}\left(\mathbf{4}_{1}\right)-\frac{2 \sqrt{3} \pi^{2}}{p^{2}+12 q^{2}}+\ldots
$$

\subsection{Properties of the A-polynomial}

The A-polynomial of a knot has a number of interesting properties. For example, as we already mentioned earlier, one can choose a normalization such that $A(l, m)$ has integer coefficients. For completeness, here we list some other basic properties of the A-polynomial that will be useful to us in the discussion below (however, one may skip this section in the first reading):

a) If $K$ is a hyperbolic knot, then $A(l, m) \neq l-1$. This already asserts that $A(l, m)$ is non-trivial for a large class of knots.

b) If $K$ is a knot in a homology sphere, then the A-polynomial involves only even powers of $m, c f$. Table 2 .

c) Reversing the orientation of $K$ does not change $A(l, m)$, but reversing the orientation of the ambient space changes $A(l, m)$ to $A(l, 1 / m)$. Note, in particular, that the A-polynomial is powerful enough to distinguish mirror knots.

d) An important property of the A-polynomial is that under the change of basis

$$
\left(\begin{array}{c}
\gamma_{l} \\
\gamma_{m}
\end{array}\right) \longrightarrow\left(\begin{array}{ll}
a & b \\
c & d
\end{array}\right)\left(\begin{array}{c}
\gamma_{l} \\
\gamma_{m}
\end{array}\right), \quad\left(\begin{array}{ll}
a & b \\
c & d
\end{array}\right) \in S L(2, \mathbb{Z})
$$

the A-polynomial transforms as

$$
A(l, m) \longrightarrow A\left(l^{d} m^{-b}, l^{-c} m^{a}\right) .
$$

e) The A-polynomial is reciprocal, i.e. $A(l, m)= \pm A(1 / l, 1 / m)$ up to powers of $l$ and $m$.

f) The A-polynomial is tempered, i.e. the faces of the Newton polygon of $A(l, m)$ define cyclotomic polynomials in one variable.

$g)$ If $K_{1}$ and $K_{2}$ are two knots and $K_{1} \# K_{2}$ is their connected sum, then $A_{K_{1} \# K_{2}}$ is divisible by $A_{K_{1}} \cdot A_{K_{2}} /(l-1)$.

h) Under some mild technical assumptions, the A-polynomial of a knot complement has a property that $A(l, \pm 1)= \pm(l-1)^{n_{+}}(l+1)^{n_{-}}$for some non-negative integers $n_{+}$ and $n_{-}$[46]. The geometric interpretation of these integer numbers is not known at present. 
i) Although it seems unlikely that the A-polynomial can be defined by iterative skein relations as, for example, the Jones or Alexander polynomials, there is a relation between the A-polynomial and the Alexander polynomial. However, the A-polynomial is a strictly stronger invariant of knots than the Alexander polynomial [46].

j) On the other hand, the A-polynomial is not a complete invariant of knots: there are different knots with the same A-polynomial, see e.g. [53]. In particular, mutants seem to have the same A-polynomial.

$k$ ) In the mathematical literature, the A-polynomial of a knot was used to extract a great deal of subtle information about the knot complement. For example, the slopes of the sides of the Newton polygon of $A(l, m)$ are boundary slopes of incompressible surfaces 9 in $M$ which correspond to ideal points of $L$.

Further properties of the A-polynomial can be found in [46,53,54].

\section{Quantization}

\subsection{A-polynomial as a Lagrangian Submanifold}

In the previous section we described the classical solutions in a $S L(2, \mathbb{C})$ Chern-Simons theory with a single Wilson loop $W_{(l, m)}(K)$ or, equivalently, the classical solutions in a theory without the Wilson loop, defined on its complement, $M=\mathbf{S}^{3} \backslash K$. In particular, we found that the classical solutions are parametrized by points on an algebraic curve $L$, which is a zero locus of the A-polynomial, $A(l, m)$. Since the knot complement $M$ is a compact 3-manifold with a single torus boundary, $\Sigma=T^{2}$, there is a natural embedding

$$
L \hookrightarrow \mathcal{P}
$$

induced by restricting a flat connection from $M$ to $\Sigma$. Here, $\mathcal{P}=\mathbb{C}^{*} \times \mathbb{C}^{*}$ is the moduli space of representations of $\pi_{1}(\Sigma)$ in $S L(2, \mathbb{C})$, parametrized by two complex numbers, $l$ and $m, c f .(2.10)$.

9 A proper embedding of a connected orientable surface $F \rightarrow M$ is called incompressible if the induced map $\pi_{1}(F) \rightarrow \pi_{1}(M)$ is injective. Its boundary slope is defined as follows. An incompressible surface $(F, \partial F)$ gives rise to a collection of parallel simple closed loops in $\partial M$. Choose one such loop and write its homology class as $\gamma_{l}^{a} \gamma_{m}^{b}$. Then, the boundary slope of $(F, \partial F)$ is defined as a rational number $a / b$. 
In the Hamiltonian approach [3], one regards $\mathcal{P}$ as a classical phase space associated to a closed surface $\Sigma$. Indeed, there is a symplectic structure $\omega$ on $\mathcal{P}$ derived from the classical Poisson bracket of gauge fields in the Chern-Simons theory (1.1). In order to see this explicitly, let us study the theory on $\Sigma \times \mathbb{R}^{1}$, i.e. near the boundary of $M$. On $\Sigma \times \mathbb{R}^{1}$ it is natural to choose the gauge $A_{0}=0$. Then, the Chern-Simons Lagrangian (1.1), which in this gauge looks like

$$
I=\frac{t}{8 \pi} \int d t \int_{\Sigma} \epsilon^{i j} \operatorname{Tr} \mathcal{A}_{i} \frac{d}{d t} \mathcal{A}_{j}+\frac{\bar{t}}{8 \pi} \int d t \int_{\Sigma} \epsilon^{i j} \operatorname{Tr} \overline{\mathcal{A}}_{i} \frac{d}{d t} \overline{\mathcal{A}}_{j}
$$

leads to the following Poisson brackets:

$$
\begin{aligned}
& \left\{\mathcal{A}_{i}^{a}(x), \mathcal{A}_{j}^{b}(y)\right\}=\frac{4 \pi}{t} \cdot \delta^{a b} \epsilon_{i j} \delta^{2}(x-y) \\
& \left\{\overline{\mathcal{A}}_{i}^{a}(x), \overline{\mathcal{A}}_{j}^{b}(y)\right\}=\frac{4 \pi}{\bar{t}} \cdot \delta^{a b} \epsilon_{i j} \delta^{2}(x-y)
\end{aligned}
$$

These relations induce a Poisson structure on the classical phase space $\mathcal{P}$, which is parametrized by the $S L(2, \mathbb{C})$ holonomies $(2.10)$ around the longitude $\gamma_{l}$ and the meridian $\gamma_{m}$. It is convenient to introduce new variables $u$ and $v$, such that

$$
l=\exp (u) \quad, \quad m=\exp (v)
$$

Since the curves $\gamma_{l}$ and $\gamma_{m}$ intersect transversely in a single point, from (3.3) we find (see also [57,51]):

$$
\begin{aligned}
& \{u, v\}=\frac{4 \pi}{t} \\
& \{\bar{u}, \bar{v}\}=\frac{4 \pi}{\bar{t}}
\end{aligned}
$$

These relations, together with $\{u, \bar{v}\}=\{\bar{u}, v\}=0$, lead to the following non-trivial Poisson brackets for the real and imaginary components of $u$ and $v$ :

$$
\begin{aligned}
& \{\operatorname{Re}(v), \operatorname{Re}(u)\}=\{\operatorname{Im}(u), \operatorname{Im}(v)\}=-2 \pi \frac{k}{k^{2}-\sigma^{2}} \\
& \{\operatorname{Re}(u), \operatorname{Im}(v)\}=-\{\operatorname{Re}(v), \operatorname{Im}(u)\}=i 2 \pi \frac{\sigma}{k^{2}-\sigma^{2}}
\end{aligned}
$$

Here we also used the explicit expression for the parameters $t=k+\sigma$ and $\bar{t}=k-\sigma$, both of which are real in the present discussion.

10 Note, that our definition of $u$ and $v$ differs from 52,55,56 by a factor of 2 . 
Writing the Poisson brackets (3.6) in the form $\left\{x^{i}, x^{j}\right\}=\omega^{i j}$ and inverting the constant skew-symmetric matrix $\omega^{i j}$, we obtain the corresponding 2 -form $\omega=\omega_{i j} d x^{i} \wedge d x^{j}$ :

$$
\omega=\frac{k}{\pi} \omega_{k}+i \frac{\sigma}{\pi} \omega_{\sigma}
$$

where $\omega_{k}$ and $\omega_{\sigma}$ are real non-degenerate 2 -forms on $\mathcal{P}$, given by

$$
\omega_{k}=d \operatorname{Re}(v) \wedge d \operatorname{Re}(u)+d \operatorname{Im}(u) \wedge d \operatorname{Im}(v)
$$

and

$$
\omega_{\sigma}=-d \operatorname{Re}(u) \wedge d \operatorname{Im}(v)+d \operatorname{Re}(v) \wedge d \operatorname{Im}(u)
$$

The differential 2-form $\omega$ is manifestly closed, $d \omega=0$. Also, notice that $\omega$ has both real and imaginary components. If its imaginary part vanishes (that is, if $\sigma \in i \mathbb{R}$ ), we can interpret (3.7) as the usual symplectic structure on the phase space $\mathcal{P}$. This system can be easily quantized by regarding $u$ and $v$ as operators on a certain Hilbert space, $\mathcal{H}_{\Sigma}$, and by replacing the Poisson brackets with commutators, $\{,\} \rightarrow i[$,$] . The resulting effective$ quantum mechanics will be discussed in more detail below.

On the other hand, if $\sigma \in \mathbb{R}$ (and, say, $k=0$ ), the Poisson structure (3.6) gives rise to an imaginary 2-form $\omega=i(\sigma / \pi) \omega_{\sigma}$, where $\omega_{\sigma}$ is the natural symplectic structure on $\mathcal{P}$. Indeed, the phase space $\mathcal{P}=\mathbb{C}^{*} \times \mathbb{C}^{*}$ can be regarded as the total space of the cotangent bundle (2.12), with the natural symplectic 2-form (3.9). However, since in (3.7) we have an imaginary multiple of this 2-form, the quantization of this system is more naturally interpreted as a Euclidean quantum mechanics. In general, if both $k$ and $\sigma$ are non-zero, we deal with a mixed situation. Notice, that this subtlety does not appear in a Chern-Simons theory with real parameter $s$, which is related to de Sitter gravity in $2+1$ dimensions. In this theory, one finds the Poisson brackets (3.6) with purely real values on the right-hand side. Therefore, a quantization of such system leads to an ordinary quantum mechanics on a "non-commutative" 2-torus, parametrized by $\operatorname{Im}(v)$ and $\operatorname{Im}(u)$.

To summarize, we found that the classical Poisson bracket in the Chern-Simons theory gives $C^{\infty}(\mathcal{P})$ the structure of a Lie algebra over $\mathbb{C}$, and suggests to interpret $(\mathcal{P}, \omega)$ as a classical Hamiltonian system. To make this interpretation even more explicit, by a linear change of variables we can always bring the 2 -form $\omega$ to the canonical form

$$
\omega=\sum_{i} d p_{i} \wedge d q_{i}
$$


where $q_{i}$ and $p_{i}, i=1,2$, are the canonical coordinates and momenta (given by linear combinations of the real and imaginary components of $u$ and $v$ ).

In Hamiltonian mechanics, a (semi-)classical state of a system is described by a Lagrangian submanifold in $\mathcal{P}$, that is a middle dimensional subvariety $L \subset \mathcal{P}$ such that the restriction of $\omega$ to $L$ vanishes,

$$
\left.\omega\right|_{L}=0
$$

In our problem, a character variety $L$ that was defined in 2.15) as the zero locus of the Apolynomial is a natural candidate for a classical state. Indeed, it is a middle dimensional subvariety in $\mathcal{P}$ which, by definition, describes the classical field configurations on the boundary $\Sigma=\partial M$ that can be extended to a 3-manifold $M$. On the other hand, $L$ is defined as a holomorphic curve in $\mathcal{P}=\mathbb{C}^{*} \times \mathbb{C}^{*}$, with respect to its natural complex structure, rather than a Lagrangian submanifold11. However, by performing a hyperKähler rotation it is easy to check that $L$ is indeed a Lagrangian submanifold, with respect to the symplectic structures (3.8) and (3.9) (and, hence, with respect to any linear combination thereof). Specifically, we can write the 2-form (3.8) as

$$
\begin{aligned}
\omega_{k} & =d \operatorname{Re}(v) \wedge d \operatorname{Re}(u)+d \operatorname{Im}(u) \wedge d \operatorname{Im}(v)= \\
& =\frac{1}{4}(d v+d \bar{v}) \wedge(d u+d \bar{u})-\frac{1}{4}(d u-d \bar{u}) \wedge(d v-d \bar{v})= \\
& =-\frac{1}{2}(d u \wedge d v+d \bar{u} \wedge d \bar{v})
\end{aligned}
$$

Clearly, this 2-form vanishes when restricted to the holomorphic curve $A\left(e^{u}, e^{v}\right)=0$. Similarly, one can check that the restriction of the 2-form (3.9) also vanishes

$$
\begin{aligned}
\omega_{\sigma} & =-d \operatorname{Re}(u) \wedge d \operatorname{Im}(v)+d \operatorname{Re}(v) \wedge d \operatorname{Im}(u)= \\
& =-\frac{1}{4 i}(d u+d \bar{u}) \wedge(d v-d \bar{v})+\frac{1}{4 i}(d v+d \bar{v}) \wedge(d u-d \bar{u})= \\
& =\frac{i}{2}(d u \wedge d v-d \bar{u} \wedge d \bar{v})
\end{aligned}
$$

when restricted to $L$. Hence, the same is true for any linear combination of $\omega_{k}$ and $\omega_{\sigma}$. In particular, the condition (3.11) holds for the complex 2-form (3.7).

We conclude that in the $S L(2, \mathbb{C})$ Chern-Simons theory the zero locus $L$ of the Apolynomial is naturally regarded as a Lagrangian submanifold in the phase space $(\mathcal{P}, \omega)$.

11 A similar situation occurs in a different, though possibly not unrelated context in string theory [58]. 
Hence, we are naturally led to the quantization of $(\mathcal{P}, \omega)$ in a real polarization 12 , where elements of quantization are associated with Lagrangian submanifolds, $c f$. [61]. Then, it is natural to expect that a quantization of this Hamiltonian system gives the quantum Hilbert space, $\mathcal{H}_{\Sigma}$, of the $S L(2, \mathbb{C})$ Chern-Simons theory in genus one. In particular, the semiclassical partition function (1.2) for a 3-manifold $M$ with a single torus boundary, $\Sigma=T^{2}$, is given by the semi-classical wave function, $Z(M) \in \mathcal{H}_{\Sigma}$, supported on the Lagrangian submanifold $L$. To be more precise, the partition function $Z(M)$ is a half-density 23 on $L$, and the resulting quantum system can be naturally viewed as a quantum mechanics on a "non-commutative" 2-torus. Below, our goal will be to explain this prescription in more detail and to verify that it leads to meaningful results.

\section{Example. The Figure-eight Knot}

As in the previous example, let $K$ be the figure-eight knot, and let $M=\mathbf{S}^{3} \backslash K$ be its complement. For a moment, let us also set $k=0$. Then, the Poisson brackets (3.6) suggest to define the canonical variables on the four-dimensional phase space $\mathcal{P}$ as

$$
\left(\begin{array}{c}
p_{1} \\
p_{2}
\end{array}\right)=\left(\begin{array}{c}
-\operatorname{Re}(u) \\
\operatorname{Re}(v)
\end{array}\right) \text { and }\left(\begin{array}{c}
q_{1} \\
q_{2}
\end{array}\right)=\left(\begin{array}{c}
\operatorname{Im}(v) \\
\operatorname{Im}(u)
\end{array}\right)
$$

In terms of these variables, the zero locus of the A-polynomial of the figure-eight knot, with the factor $(l-1)$ included, is given by

$$
\left(\cosh \left(4 p_{2}+4 i q_{1}\right)-\cosh \left(2 p_{2}+2 i q_{1}\right)-\cosh \left(p_{1}-i q_{2}\right)-1\right)\left(e^{i q_{2}-p_{1}}-1\right)=0
$$

This complex equation defines two real constraints in a dynamical system with trivial Hamiltonian, whose quantization leads to an effective quantum mechanics on a torus, parametrized by $q_{1}$ and $q_{2}$. When the parameter $s$ in the Chern-Simons action (1.1) is real, one finds ordinary quantum mechanics, whereas for imaginary values of $s$ (i.e. for $s=-i \sigma)$ the resulting system is best described as a Euclidean quantum mechanics. In both cases, turning on the 'level' $k$ leads to a deformation of the Poisson structure (3.6), which can be naturally interpreted as a non-commutativity of the torus coordinates, $q_{1}$ and $q_{2}$ (as well as momenta, $p_{1}$ and $p_{2}$ ).

12 For quantization of Chern-Simons theory in complex polarizations see [1, 59, 60, 37].

13 Given an $n$-dimensional manifold $X$, let $\mathcal{B} X$ denote the frame bundle of $V=T X$; its structure group is $G L(V)$. Then, an $r$-density on $X$ is a smooth function $f: \mathcal{B} X \rightarrow \mathbb{C}$ which transforms under the action of $G L(V)$ as $f \circ g=|\operatorname{Det}(g)|^{r} f$, where $g \in G L(V)$ and $\operatorname{Det}(g)$ is the determinant of $g$. For $r=1$ this definition gives the usual density on $X$, and for $r=1 / 2$ it gives a half-density on $X$. 
Before we proceed, let us briefly point out that the Chern-Simons action induces $S L(2, \mathbb{C}) / S U(2)$ WZW theory on the boundary manifold $\Sigma$. However, the structure of the Hilbert space in this theory is not completely clear at present. One might hope to get further insights from the present approach.

\subsection{Semi-Classical Approximation}

Following the general prescription formulated in the end of the previous subsection, here we study the semi-classical behavior of the partition function $Z(M)$ by quantizing the Hamiltonian system associated with $(\mathcal{P}, \omega)$ and a Lagrangian submanifold $L$. As in the standard classical mechanics, we introduce a canonical 1-form (also known as a Liouville form), which in the canonical variables $\left(p_{i}, q_{j}\right)$ can be written as

$$
\theta=\sum_{i} p_{i} d q_{i}
$$

We note that this 1-form may not be globally defined; this happens, for example, when the phase space is compact. However, locally we can always write $\omega=d \theta$, and because $\left.\omega\right|_{L}=0$ holds by the definition of the Lagrangian submanifold $L$, we find that the restriction of the canonical 1-form to $L$ is closed,

$$
\left.d \theta\right|_{L}=0
$$

The cohomology class in $H^{1}(L, \mathbb{R})$ induced by the 1 -form $\theta$ is called the Liouville class of the Lagrangian immersion $L \hookrightarrow \mathcal{P}$. Lagrangian immersions for which the Liouville class is trivial are called exact Lagrangian immersions.

Consistent quantization of $(\mathcal{P}, \omega)$ with a Lagrangian submanifold $L$ requires the Liouville class of $L$ to obey certain conditions. In order to explain these conditions, let us proceed with the WKB quantization and define a phase function $S$, usually called the action integral, such that

$$
d S=\left.\theta\right|_{L}
$$

Then, the semi-classical approximation to the wave function supported on the Lagrangian submanifold $L$ is given by

$$
Z \simeq \sum_{\alpha} \psi \cdot e^{i S / \hbar} e^{i \pi \eta / 4}+O(\hbar)
$$


where the sum is over a discrete, finite set of the components of $L, \eta$ is the famous Maslov correction to the phase of the semi-classical wave function, and the amplitude $\psi$ is a halfdensity on $L$, which obeys the transport equation [62,63]:

$$
\mathcal{L} \psi=0
$$

In order to understand the meaning of each term in (3.19), it is instructive to consider a finite dimensional integral

$$
Z=\int \prod_{i} d q_{i} e^{i S\left(q_{i}\right) / \hbar}
$$

In the stationary phase approximation, this integral is dominated by the contribution from the critical points,

$$
Z \simeq \sum_{\alpha} \frac{e^{i S / \hbar}}{\sqrt{\operatorname{det}(-i \cdot \operatorname{Hess}(S))}}+O(\hbar)
$$

where $\operatorname{Hess}(S)$ stands for the Hessian of the phase function $S$. Here, a phase of the square root of the determinant requires extra care. Namely, if $S$ is a real function, $\operatorname{Hess}(S)$ has only real eigenvalues, $\lambda_{i}$. Each positive eigenvalue contributes $(-i)^{-1 / 2}=e^{i \pi / 4}$ to the phase of the above expression, whereas each negative eigenvalue contributes $i^{-1 / 2}=e^{-i \pi / 4}$. Therefore, a refined version of the formula (3.22) looks like:

$$
Z \simeq \sum_{\alpha} e^{i S / \hbar} e^{i \pi \eta / 4}|\operatorname{det}(\operatorname{Hess}(S))|^{-1 / 2}+O(\hbar)
$$

where the $\eta$-invariant

$$
\eta=\sum_{i} \operatorname{sign} \lambda_{i}
$$

is defined as the (regularized) signature of the $\operatorname{Hessian}, \operatorname{Hess}(S)$, that is the total number of positive eigenvalues of $\operatorname{Hess}(S)$ minus the total number of negative eigenvalues of $\operatorname{Hess}(S)$.

Now, let us return to the semi-classical wave function (3.19) associated with a Lagrangian submanifold $L$. Notice, that when the restriction of $\theta$ to $L$ is an exact 1-form, the phase function $S=\int \theta$ is well defined. However, if the Lioville class of $\theta$ is non-trivial, the phase function $S$ depends on the choice of the integration path in $L$. In particular, a difference between two such choices will change the action integral by a period of $\theta$,

$$
\Delta S=\oint_{C} \theta
$$


where $C$ is a closed 1-cycle on $L$. In order for the semi-classical expression (3.19) to be unambiguously defined, all the periods of $\theta$ must be integer multiples of $2 \pi \hbar$, for some value of $\hbar$ :

$$
\frac{1}{2 \pi \hbar} \oint_{C} \theta \in \mathbb{Z} \quad, \quad \forall C \in \pi_{1}(L)
$$

A Lagrangian submanifolds $L \hookrightarrow \mathcal{P}$ which obeys this condition is called quantizable, and the corresponding values of $\hbar$ for which (3.25) holds are called admissible for $L$. Notice, that the set of quantizable Lagrangian submanifolds with large first Betti number is usually rather limited.

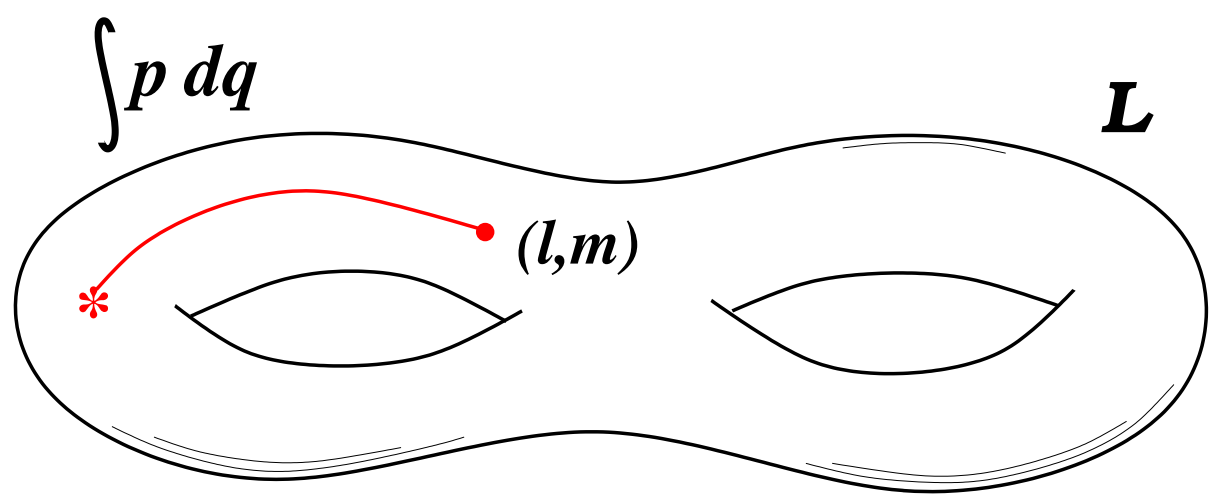

Fig. 7: The moduli space of the $S L(2, \mathbb{C})$ Chern-Simons theory on a knot complement is described by the A-polynomial of the knot. The action integral of the corresponding quantum mechanics can be obtained by integrating $\theta=\sum_{i} p_{i} d q_{i}$ over a path on the curve $A(l, m)=0$.

Now, let us apply this to our problem. We set $\hbar=1$; the role of the Planck constant is played by $k^{-1}$ and $\sigma^{-1}$, so that the semi-classical limit corresponds to $k, \sigma \rightarrow \infty$. Furthermore, from the defining equations (3.7) and (3.16) we obtain a canonical 1-form,

$$
\theta=\frac{k}{\pi} \theta_{k}+i \frac{\sigma}{\pi} \theta_{\sigma}
$$

where $\theta_{k}$ and $\theta_{\sigma}$ are real 1 -forms,

$$
\theta_{k}=\operatorname{Re}(v) d \operatorname{Re}(u)+\operatorname{Im}(u) d \operatorname{Im}(v)
$$

and

$$
\theta_{\sigma}=-\operatorname{Re}(u) d \operatorname{Im}(v)+\operatorname{Re}(v) d \operatorname{Im}(u)
$$


Since the 1-form $\theta$ is complex-valued, the condition for $L$ to be quantizable implies two independent sets of constraints corresponding, respectively, to the imaginary and real parts of the Bohr-Sommerfeld condition (3.25):

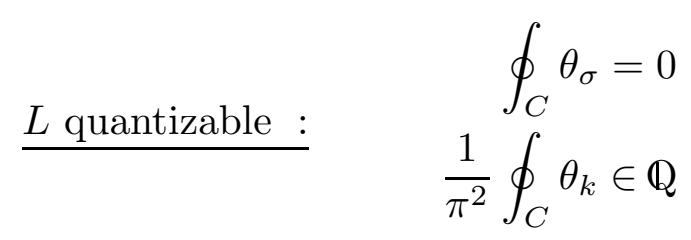

Provided that these two conditions are satisfied for any 1-cycle $C \subset L$, the system can be consistently quantized and one can write the semi-classical expression for the partition function in the form (3.19). The first condition in (3.29) asserts that all the periods of $\theta_{\sigma}$ must vanish. Equivalently, $\theta_{\sigma}$ should be an exact 1-form, when restricted to $L$.

It turns out that, for a Lagrangian submanifold $L$ defined as a zero locus of the Apolynomial, the restriction of $\theta_{\sigma}$ to $L$ is always an exact 1-form. Specifically, using a classical formula of Schläfli concerning the volume of infinitesimally deformed polyhedra, one can show that

$$
\left.\theta_{\sigma}\right|_{L}=\frac{1}{2} d \operatorname{Vol}(M)
$$

where $\operatorname{Vol}(M)$ is the volume of the hyperbolic 3-manifold $M$, which in the present discussion is a complement of a knot $K$. This important result is due to Hodgson [64] with improvements by Dunfield [65]. Similarly, the real part of the 1-form $\theta$ is related to the Chern-Simons invariant of the 3-manifold $M$,

$$
\left.\theta_{k}\right|_{L}=-\pi^{2} d C S(M)
$$

This formula was originally conjectured by Neumann and Zagier [52], and later proved by Yoshida [55, see also 66,56].

From the equations (3.30) and (3.31) it follows that imaginary and real components of the action integral $S$ are related, respectively, to the volume and the Chern-Simons invariant of the knot complement $M$ :

$$
S=\frac{i \sigma}{2 \pi} \operatorname{Vol}(M)-\pi k C S(M)
$$

This is indeed the expected semi-classical behavior of the $S L(2, \mathbb{C})$ Chern-Simons action (1.7). We can also write it as

$$
d S=t d F+\bar{t} d \bar{F}
$$


where

$$
d F=\frac{1}{4 \pi}(v d u-u d v+d(u \bar{v}))
$$

Combining the formulas (3.19) and (3.32) together, we obtain:

$$
Z(M) \simeq \sum_{\alpha} \psi \cdot e^{i \pi \eta / 4} \cdot \exp \left(-\frac{\sigma}{2 \pi} \operatorname{Vol}(M)-i \pi k C S(M)\right)+\ldots
$$

This is the usual expression for the semi-classical wave function in a quantum mechanics with the action integral (3.32). Let us now compare this result with a general form of the (unnormalized) semi-classical partition function in a Chern-Simons theory [3]:

$$
Z(M) \simeq \sum_{\alpha} \frac{\operatorname{det}(\Delta)}{\sqrt{\left|\operatorname{det}\left(L_{-}\right)\right|}} e^{i \pi \eta\left(\mathcal{A}^{(\alpha)}\right) / 4} \cdot e^{i I\left(\mathcal{A}^{(\alpha)}\right)}+\ldots
$$

where $\Delta$ is the standard Laplacian and $L_{-}$denotes a restriction of the self-adjoint operator $L=* D+D *$ to the space of odd forms on $M$ (not to be confused with the character variety $L)$. For a Chern-Simons theory with complex gauge group one further has [1,67]:

$$
\eta\left(\mathcal{A}^{(\alpha)}\right)=0
$$

It is clear that our result (3.34) is very similar to (3.35). In fact, one can easily see the terms in both expression which have the same origin and, therefore, should be identified. For example, each critical point in the quantum mechanics problem corresponds to a flat $S L(2, \mathbb{C})$ connection, $\mathcal{A}^{(\alpha)}$, in the Chern-Simons theory. Among these flat connections, there is a geometric one, associated with the hyperbolic structure on the knot complement $M$. The Chern-Simons action, $I\left(\mathcal{A}^{\text {(hyperb) }}\right)$, of this flat connection is given by (3.32), and it was already discussed in this section. Moreover, $\eta\left(\mathcal{A}^{(\alpha)}\right)$ should be identified with the Maslov correction to the phase of the semi-classical expression (3.34), and (3.36) implies that this correction vanishes. This should also follow directly from the properties of the A-polynomial. Finally, the absolute value of the ratio of the determinants in (3.35), which is often called the Reidemeister-Ray-Singer torsion, $T\left(\mathcal{A}^{(\alpha)}\right)$,

$$
\sqrt{T\left(\mathcal{A}^{(\alpha)}\right)}=\frac{\operatorname{det}(\Delta)}{\sqrt{\left|\operatorname{det}\left(L_{-}\right)\right|}}
$$

is related to the amplitude, $\psi$, of the quantum wave function (3.34).

Notice that, besides the critical point corresponding to the hyperbolic $S L(2, \mathbb{C})$ connection, the sum (3.34) also contains terms corresponding to other flat connections. Even 
though the Chern-Simons action, $I\left(\mathcal{A}^{(\alpha)}\right)$, of these flat connections is given by (3.32), where the "volume" and the "Chern-Simons invariant" can be computed by integrating the 1-forms (3.27) and (3.28) over different branches of the zero locus of the A-polynomial, they no longer have a nice geometric interpretation. For example, there are flat connections with negative "volume". In particular, if $K$ is a hyperbolic knot, then the semi-classical behavior of the partition function (3.35) is dominated by the flat connection whose volume is equal to minus the hyperbolic volume of $M$,

$$
Z(M) \simeq \sqrt{T\left(\mathcal{A}^{(\text {hyperb })}\right)} \exp \left(\frac{\sigma}{2 \pi} \operatorname{Vol}(M)+i \pi k C S(M)\right)+\ldots
$$

\subsection{Perturbation Theory}

Using effective quantum mechanics, we studied the semi-classical limit of the partition function, $Z(M)$, in the $S L(2, \mathbb{C})$ Chern-Simons theory on a knot complement, $M=\mathbf{S}^{3} \backslash K$. Equivalently, we may also think of $Z(M)$ as the (unnormalized) expectation value of a Wilson line, $W_{(l, m)}(K)$, in an infinite-dimensional representation $R$ of $S L(2, \mathbb{C})$. In either interpretation, it would be interesting to extend this analysis beyond the leading order and to compute the higher-order perturbative corrections to the partition function (3.34). General arguments suggest that the result should be in the form

$$
Z \simeq \sum_{\alpha} \sqrt{T\left(\mathcal{A}_{\alpha}\right)} e^{i I\left(\mathcal{A}^{(\alpha)}\right)} \times\left(1+\sum_{m, n=1}^{\infty} \frac{b_{m, n}(\alpha)}{k^{m} \sigma^{n}}\right)
$$

where the coefficients $b_{m, n}(\alpha)$ are expected to be related to some perturbative invariants of a (decorated) knot $K$, analogous to the Vassiliev invariants, $c f$. «4,68.

There are two important remarks that one should bear in mind. First, a calculation of the coefficients $b_{m, n}(\alpha)$ requires a perturbative computation of the Feynman diagrams in the background of a non-trivial flat connection $\mathcal{A}^{(\alpha)}$. The second remark has to do with the fact that $R$ is an infinite-dimensional representation of $S L(2, \mathbb{C})$. In fact, if $R$ was merely a complexification of a finite-dimensional $S U(2)$ representation, then the coefficients $b_{m, n}(\alpha)$ would be exactly the same as in the $S U(2)$ Chern-Simons theory, and nothing new could be gained [4]. 


\subsection{Non-perturbative Aspects}

We found the semi-classical expression for the partition function $Z(M)$ and briefly discussed higher-order perturbative corrections. However, using the effective quantum mechanics one might hope to compute the entire series $Z(M)$, say, using the technique of the geometric quantization [59, 60, 61, 62, 63]. The semi-classical result (3.34) is simply the leading order approximation to the exact expression for $Z(M)$, which obeys

$$
\widehat{A} Z=0
$$

In quantum theory, this operator equation represents the classical constraint $A(l, m)=0$,

cf. e.g. (3.15). Namely, the operator $\widehat{A}$ is obtained from the A-polynomial by replacing $l$ and $m$ with the corresponding operators, $\widehat{l}$ and $\widehat{m}$, whose commutation relations follow from (3.5). We also note that $Z(M)$ automatically satisfies the Hamiltonian constraint, $\widehat{H} Z=0$, which can be naturally interpreted as the Wheeler-DeWitt equation (1.11) in three-dimensional Euclidean gravity.

As we explain in the later sections, the asymptotic behavior of the colored Jones polynomial is very similar to the perturbative expansion (3.38) - (3.39). This suggests a close connection between the exact, non-perturbative partition function of the $S L(2, \mathbb{C})$ Chern-Simons theory and a certain analytic continuation of the colored Jones polynomial,

$$
Z(M) \stackrel{?}{\longrightarrow} J_{N}(K, q)
$$

In particular, it is natural to speculate that the colored Jones polynomial is related to the $S L(2, \mathbb{C})$ partition function when the variable $q$ is not a root of unity, $c f$. Table 1 . A further evidence for this relation comes from the recent work [69], where it was shown that the colored Jones polynomial for certain knots obeys the $q$-difference equation (3.40). We postpone further study of the expected relation (3.41) to future work.

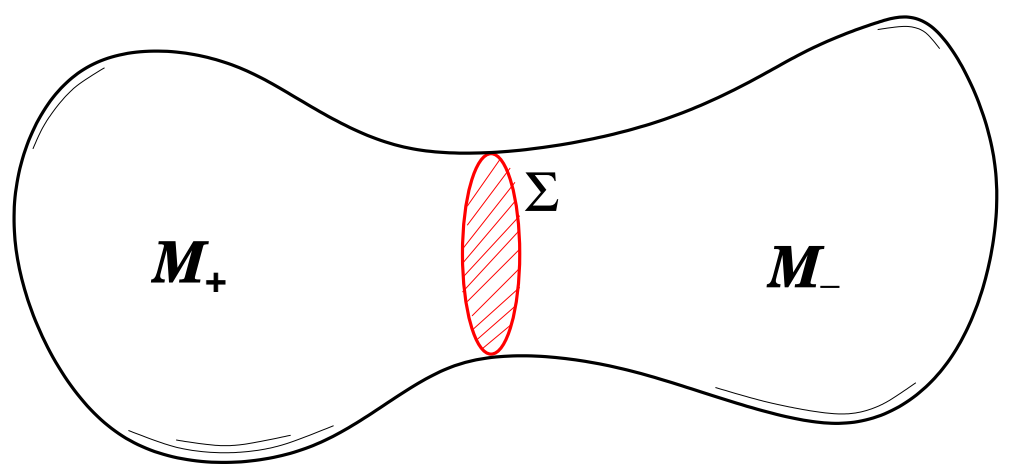

Fig. 8: The manifold $M$ is a connected sum of the 3 -manifolds $M_{+}$and $M_{-}$, joined along their common boundary $\Sigma$. 
The non-perturbative partition function $Z(M)$ has a number of nice properties that follow directly from the path integral formulation (1.4). Suppose, for example, that $M$ is a connected sum of the 3-manifolds, $M_{ \pm}$, joined along the common sphere boundary $\Sigma=\mathbf{S}^{2}$ (see fig. 8):

$$
M=M_{+} \cup_{\Sigma} M_{-}
$$

Then, the Chern-Simons partition function $Z(M)$ is given by

$$
Z(M)=\frac{Z\left(M_{+}\right) \times Z\left(M_{-}\right)}{Z\left(\mathbf{S}^{3}\right)}
$$

where $Z\left(\mathbf{S}^{3}\right)$ denotes the partition function for a 3 -sphere.

Similarly, one can compute $Z(M)$ for a homology 3-sphere $M$ represented as a union (3.42) of $M_{+}$and $M_{-}$joined along a common torus boundary, $\Sigma=T^{2}$. Following the discussion in section 2 , to each $M_{ \pm}$we can associate a representation variety (the zero locus of the corresponding A-polynomial):

$$
L_{ \pm}=\operatorname{Hom}\left(\pi_{1}\left(M_{ \pm}\right) ; S L(2, \mathbb{C})\right) / S L(2, \mathbb{C})
$$

Moreover, since $M_{ \pm}$are 3-manifolds with a common torus boundary $\Sigma=T^{2}$, the inclusions of $\Sigma$ into $M_{ \pm}$induce the embeddings:

$$
L_{ \pm} \hookrightarrow \mathbb{C}^{*} \times \mathbb{C}^{*}=\operatorname{Hom}\left(\pi_{1}(\Sigma) ; S L(2, \mathbb{C})\right) / S L(2, \mathbb{C})
$$

The intersection points of $L_{+}$and $L_{-}$are in one-to-one correspondence with the conjugacy classes of representations of the fundamental group $\pi_{1}(M)$ into $S L(2, \mathbb{C})$. Therefore, we expect that the partition function

$$
Z(M)=\left\langle Z\left(M_{+}\right) \mid Z\left(M_{-}\right)\right\rangle
$$

is dominated by the contribution from the intersection points of $L_{+}$and $L_{-}$.

The above discussion is reminiscent of the definition of the Casson invariant [70, [1]. Indeed, using the A-polynomial we can define (and in many cases easily compute) the $S L(2, \mathbb{C})$ Casson invariant of $M$ as the weighted sum of the intersection points of $L_{+}$and $L_{-}$inside $\mathbb{C}^{*} \times \mathbb{C}^{*}$

$$
\lambda_{S L(2, \mathbf{C})}(M)=\#\left(L_{+} \cap L_{-}\right)
$$




\section{Example. $M=\mathbf{S}^{3}$}

Consider $M=\mathbf{S}^{3}$ with the standard Heegard splitting, $M=M_{+} \cup_{\Sigma} M_{-}$, where $\Sigma=T^{2}$ and each $M_{ \pm}$is a copy of the solid torus. Since the boundaries of $M_{ \pm}$are glued together with a relative $S L(2, \mathbb{Z})$-transformation,

$$
\left(\begin{array}{c}
\gamma_{l} \\
\gamma_{m}
\end{array}\right) \longrightarrow\left(\begin{array}{cc}
0 & -1 \\
1 & 0
\end{array}\right)\left(\begin{array}{c}
\gamma_{l} \\
\gamma_{m}
\end{array}\right)
$$

from (2.16) and (2.22) we get:

$$
L_{+}=l-1 \quad, \quad L_{-}=m-1
$$

Clearly, the curves $L_{+}$and $L_{-}$intersect at a single point, $m=l=1$, so that we find the $S L(2, \mathbb{C})$ Casson invariant $\lambda_{S L(2, \mathbf{C})}\left(\mathbf{S}^{3}\right)=1$.

It would be interesting to find a realization of the $S L(2, \mathbb{C})$ Casson invariant in ChernSimons theory with fermionic symmetries, $c f$. [16].

Finally, let us point out that throughout the paper we mainly focus on the simplest case of a single torus boundary, or a single Wilson loop $W_{(l, m)}(K)$. It would be interesting to extend this discussion to links with several components and three-manifolds with arbitrary boundary $\Sigma$ (such generalizations will be briefly discussed in the next section). In a special case, when a link consists of $r$ unlinked components $\gamma_{k}$, we have:

$$
\frac{Z\left(\mathbf{S}^{3} ; \gamma_{1}, R_{1}, \ldots, \gamma_{r}, R_{r}\right)}{Z\left(\mathbf{S}^{3}\right)}=\prod_{k=1}^{r} \frac{Z\left(\mathbf{S}^{3} ; \gamma_{k}, R_{k}\right)}{Z\left(\mathbf{S}^{3}\right)}
$$

Suppose that $r=2$ and $R_{1}=R_{2}$, i.e. we have two oriented unlinked components, $\gamma_{1}$ and $\gamma_{2}$, carrying the same representation $R$. Then, from the path integral formulation (1.4), it follows that the partition function for the connected sum $\gamma_{1} \# \gamma_{2}$ is related to the product of the partition functions for the individual link components,

$$
Z\left(\mathbf{S}^{3} ; \gamma_{1} \# \gamma_{2}, R\right)=\frac{Z\left(\mathbf{S}^{3} ; \gamma_{1}, R\right) \times Z\left(\mathbf{S}^{3} ; \gamma_{2}, R\right)}{Z\left(\mathbf{S}^{3} ; \text { unknot }, R\right)}
$$

This factorization formula also follows from the property $g$ ) of the A-polynomial listed in section 2.3 . 


\section{Topological Quantum Field Theory and Invariants of Three-Manifolds}

As was already pointed out earlier, the definition of the Chern-Simons path integral (1.4) is independent on the choice of metric on $M$, even in the quantum theory. Hence, the Chern-Simons partition function $Z\left(M ; \gamma_{i}, R_{i}\right)$ must be a topological invariant. This is not so obvious from the mathematical point of view because Chern-Simons theory with complex gauge group operates on an infinite-dimensional Hilbert space and, therefore, does not fit into the standard definition of topological quantum field theory of Atiyah and Segal [72]. Let us remind that, according to [72], a topological quantum field theory is a functor $Z$, such that: $(i)$ to each compact oriented 2-dimensional manifold $\Sigma$ without boundary one associates a finite dimensional complex vector space $\mathcal{H}_{\Sigma}$; and (ii) a compact oriented 3-dimensional smooth manifold $M$ with $\partial M=\Sigma$ determines a vector $Z(M) \in \mathcal{H}_{\Sigma}$. Moreover, $Z$ must satisfy the following axioms:

(1) We denote by $-\Sigma$ the surface $\Sigma$ with the opposite orientation. Then, we have $\mathcal{H}_{-\Sigma}=$ $\mathcal{H}_{\Sigma}^{*}$ where $\mathcal{H}_{\Sigma}^{*}$ is the dual of $\mathcal{H}_{\Sigma}$ as a complex vector space.

(2) For a disjoint union $\Sigma_{1} \sqcup \Sigma_{2}$ we have $\mathcal{H}_{\Sigma_{1} \sqcup \Sigma_{2}}=\mathcal{H}_{\Sigma_{1}} \otimes \mathcal{H}_{\Sigma_{2}}$.

(3) For the composition of cobordism迎 $\partial M_{1}=\left(-\Sigma_{1}\right) \sqcup \Sigma_{2}$ and $\partial M_{2}=\left(-\Sigma_{2}\right) \sqcup \Sigma_{3}$, the relation $Z\left(M_{1} \cup M_{2}\right)=Z\left(M_{2}\right) \circ Z\left(M_{1}\right)$ holds, where the right-hand side stands for the composition of linear maps $Z\left(M_{1}\right): \mathcal{H}_{\Sigma_{1}} \rightarrow \mathcal{H}_{\Sigma_{2}}$ and $Z\left(M_{2}\right): \mathcal{H}_{\Sigma_{2}} \rightarrow \mathcal{H}_{\Sigma_{3}}$.

(4) For an empty set $\emptyset$ we have $Z(\emptyset)=\mathbb{C}$.

(5) Let $I$ denote the closed unit interval. Then, $Z(\Sigma \times I)$ is the identity map as a linear transformation of $\mathcal{H}_{\Sigma}$.

It is easy to check that all of these conditions are satisfied in a Chern-Simons theory with compact gauge group $G$. Similarly, many of these properties extend to a theory with the complexified gauge group $G_{\mathbb{C}}$, essentially due to the path integral formulation (1.2). There is one important subtlety, however, related to the fact that in the latter theory the phase space

$$
\mathcal{P}=\operatorname{Hom}\left(\pi_{1}(\Sigma) ; G_{\mathbb{C}}\right) / G_{\mathbb{C}}
$$

is no longer compact. Indeed, the space $\mathcal{P}$ is isomorphic to the total space of the cotangent bundle, $c f$. (2.12):

$$
\mathcal{P}=T^{*} \mathcal{M}
$$

14 It follows from the axioms (1) and (2) that a compact oriented 3-manifold $M$ with $\partial M=$ $\left(-\Sigma_{1}\right) \sqcup \Sigma_{2}$ determines a linear map $Z(M) \in \operatorname{Hom}_{\mathbf{C}}\left(\mathcal{H}_{\Sigma_{1}}, \mathcal{H}_{\Sigma_{2}}\right)$. Such a manifold $M$ is called a cobordism between $\Sigma_{1}$ and $\Sigma_{2}$. 
where $\mathcal{M}$ is a representation space of $\pi_{1}(\Sigma)$ into the compact part of the gauge group, $G$. Therefore, the corresponding Hilbert space $\mathcal{H}_{\Sigma}$ is not finite dimensional. To be more specific, by analogy with the $S L(2, \mathbb{C})$ case studied in this paper, one might define the quantum Hilbert space $\mathcal{H}_{\Sigma}$ of a Chern-Simons theory with gauge group $G_{\mathbb{C}}$ as a space of half-densities on $\mathcal{P}$. Since this space is infinite-dimensional, one needs a refinement of the above definition in order to prove that we deal with a topological quantum field theory in a mathematical sense. Physical considerations suggest, however, that there exists a rigorous mathematical definition of the topological invariant $Z\left(M ; \gamma_{i}, R_{i}\right)$, which we postpone to future work.

\section{Implications for Representation Varieties and the A-polynomial}

Apart from defining a topological invariant of three-manifolds with links colored by infinite-dimensional representations, Chern-Simons theory can also shed some light on the properties of representation varieties (2.3), in particular, on the properties of the Apolynomial. Thus, a lot of interesting information about $L=\operatorname{Hom}\left(\pi_{1}(M) ; G_{\mathbb{C}}\right) / G_{\mathbb{C}}$ and about the 3-manifold $M$ itself can be obtained by treating $L$ as a Lagrangian submanifold in $\mathcal{P}$. In particular, as we saw earlier, the Lagrangian inclusion $L \hookrightarrow \mathcal{P}$ is a natural starting point for quantization, and can lead to new connections between three-dimensional topology, symplectic geometry, and perhaps even Langlands' program [73].

For instance, it directly follows from this new perspective that the "volume formula" (3.30) and the "Chern-Simons formula" (3.31) can be generalized to hyperbolic 3-manifolds with arbitrary boundary $\Sigma$, not necessarily connected. There is a nice class of examples of such manifolds called convex cores, which have finite volume [74 and may be useful in this context. Even though the explicit description of the representation variety

$$
L=\operatorname{Hom}\left(\pi_{1}(M) ; S L(2, \mathbb{C})\right) / S L(2, \mathbb{C})
$$

may be very complicated for a generic 3-manifold $M$ with boundary $\Sigma$, the ambient space $\mathcal{P}$ is always a symplectic space of the form (4.2). Specifically, in the case we are considering, the space $\mathcal{P}$ is the total space of the cotangent bundle over the moduli space, $\mathcal{M}$, of flat $S U(2)$ connections on $\Sigma$. Let $\omega_{\sigma}$ denote the natural symplectic structure on $\mathcal{P}=T^{*} \mathcal{M}$, and let $\theta_{\sigma}$ be the corresponding canonical 1-form, such that $\omega_{\sigma}=d \theta_{\sigma}$. Then, the physical considerations suggest that

$$
\left.\theta_{\sigma}\right|_{L}=\frac{1}{2} d \operatorname{Vol}(M)
$$


should hold for arbitrary $M$ (along with a similar expression for the Chern-Simons invariant of $M)$. Furthermore, the 1-form $\theta_{\sigma}$ should be exact, when restricted to the Lagrangian submanifold $L \hookrightarrow \mathcal{P}$,

$$
\oint_{C} \theta_{\sigma}=0 \quad, \quad \forall C \in \pi_{1}(L)
$$

There is a similar set of constraints that follows from the rationality of the periods of $\theta_{k}$, cf. (3.29). Altogether, these conditions assert that $L$ is a quantizable submanifold and impose severe restrictions on its geometry, especially when $\pi_{1}(L)$ is large.

For example, these constraints lead to some non-trivial obstructions to a polynomial arising as the A-polynomial of a knot. Namely, if $\Sigma=T^{2}$ and $L$ is described by the zero locus of the A-polynomial, from (3.29) we find that the integral of the 1 -form $\theta_{\sigma}$ around any closed loop $C$ on the curve $L$ must vanish,

$$
\oint_{C} \log |l| d(\arg m)-\log |m| d(\arg l)=0
$$

and, furthermore, that the integral of the 1 -form $\theta_{k}$ must be a rational number,

$$
\frac{1}{\pi^{2}} \oint_{C} \log |m| d \log |l|+(\arg l) d(\arg m) \in \mathbb{Q}
$$

In particular, this gives the answer to a question posed by Cooper and Long in [53, 54]: "Which affine curves $L$ in $\mathbb{C}^{*} \times \mathbb{C}^{*}$ satisfy the condition that $\theta_{\sigma}$ is exact on $L$ ?" Namely, the 1 -form $\theta_{\sigma}$ should be interpreted as an "imaginary part" of the Lioville form (3.26), and then the condition (4.5) is simply the condition for $L$ to be a quantizable Lagrangian submanifold in $\mathbb{C}^{*} \times \mathbb{C}^{*}$. This suggests a further relationship to symplectic geometry. The first of the above conditions can be understood as a consequence of the Schäfli-like formula (3.30), and has a number of applications, see e.g. [53]. To the best of our knowledge, the second condition has not been discussed in the mathematical literature.

\section{Example.}

Following [53], let us demonstrate how the condition (4.5) can be used to constrain the form of the A-polynomial. For example, in Table 2 one finds the A-polynomial of the figure-eight knot:

$$
A(l, m)=-2+m^{4}+m^{-4}-m^{2}-m^{-2}-l-l^{-1}
$$

A slight modification of this polynomial gives

$$
f(l, m)=-2+m^{6}+m^{-6}-m^{2}-m^{-2}-l-l^{-1}
$$

which is not the A-polynomial of any knot since the 1 -form $\theta_{\sigma}$ has non-vanishing periods on $f(l, m)=0$, therefore, violating (4.5). However, $f(l, m)$ does exhibit every other property of the A-polynomial. It would be interesting to invent similar examples which satisfy the first condition (4.5), but fail (4.6). 
Notice, that the genus of the curve $A(l, m)=0$ grows very rapidly with the complexity of the knot. (One can get a general impression, say, by looking at the examples listed in Table 2.) Therefore, the number of non-trivial constraints in (4.5) and (4.6) also becomes very large, especially for polynomials of large degree. In fact, one might wonder if these constraints, together with the ones mentioned in section 2.3, give a complete list:

Question: Is every integral, reciprocal, and tempered polynomial $f(l, m)$ that satisfies both conditions $(4.5)$ and $(4.6)$ is the A-polynomial of some knot?

\section{A Generalization of the Volume Conjecture}

In this section 15 we show how the above approach can be used to extend the volume conjecture to incomplete hyperbolic structures on knot complements $1 \mathbf{E}$. The generalized volume conjecture, then, can be interpreted as a relation between the A-polynomial and the the colored Jones polynomial. Furthermore, in the next section we discuss a similar generalization of the Melvin-Morton-Rozansky conjecture, also inspired by physics.

\subsection{A Brief Review of the Volume Conjecture}

A well-known invariant of knots is the Jones polynomial $J(K, q)$ [78. Here we consider a more general invariant, the so-called $N$-colored Jones polynomial $J_{N}(K, q)$, associated with an $N$-dimensional irreducible representation of $S U(2)$. Thus, the usual Jones polynomial appears as a special case, $J(K, q)=J_{2}(K, q)$. Like the ordinary Jones polynomial, $J_{N}(K, q)$ can be defined by skein relations and is a Laurent polynomial in the variable $q^{1 / 2}$. Remarkably, there is a relation between the colored Jones polynomial of a knot and the volume of its complement.

The first observation along these lines was made by Kashaev, who introduced a link invariant associated with quantum dilogarithm [34]. The Kashaev's invariant assocated with a knot $K$ - usually denoted by $\langle K\rangle_{N}$ - is based on the theory of quantum dilogarithms at the $N$-th root of unity, $q=\exp (2 \pi i / N)$. Moreover, it was noticed in 34 that for certain knots the asymptotic behavior of this invariant is related to the volume of the knot complement

$$
\lim _{N \rightarrow \infty} \frac{\log \left|\langle K\rangle_{N}\right|}{N}=\frac{1}{2 \pi} \operatorname{Vol}(M)
$$

15 The work presented in this section originated from discussions with K. Krasnov.

16 See also [75, 76, 77] for a previous work in this direction. 
A generalization of this relation to all knots is known as the volume conjecture [34].

Later, it was realized in [35] that the Kashaev's invariant is related to the colored Jones polynomial evaluated at the special value of $q$ :

$$
q=e^{2 \pi i / N}
$$

Therefore, the volume conjecture can be reformulated as a relation between the volume of the knot complement and a special limit of the colored Jones polynomial:

\section{The Volume Conjecture:}

$$
\lim _{N \rightarrow \infty} \frac{\log \left|J_{N}\left(K, e^{2 \pi i / N}\right)\right|}{N}=\frac{1}{2 \pi} \operatorname{Vol}(M)
$$

In this form, the volume conjecture has been verified in a number of examples. In particular, Kashaev and Tirkkonen [79] proved that it is true for all torus knots, in a sense that the limit (5.3) is zero.

The volume conjecture was extended further in [36], where it was shown that for a large class of knots one can remove the absolute value in (5.3), so that the following limit holds 17 :

$$
\lim _{N \rightarrow \infty} \frac{\log J_{N}\left(K, e^{2 \pi i / N}\right)}{N}=\frac{1}{2 \pi}\left(\operatorname{Vol}(M)+i 2 \pi^{2} C S(M)\right)
$$

This version of the volume conjecture - which relates the asymptotic behavior of $J_{N}(K, q)$ to the volume and Chern-Simons invariant of the knot complement - is the one we are going to use here. For more work on the volume conjecture see e.g. [77, 80, 81, 82].

\subsection{The A-Polynomial and the Generalized Volume Conjecture}

Incomplete hyperbolic structures on knot complements come in continuous families. For example, if $K$ is a hyperbolic knot in the 3 -sphere, then the moduli space of hyperbolic metrics on $M=\mathbf{S}^{3} \backslash K$ has at least one component of complex dimension one. Apart from a special point representing the cusped 3-manifold, a generic point in this moduli space corresponds to an incomplete hyperbolic structure on $M$ with a conical singularity or some other kind of degeneration along the knot $K$, see e.g. [64] for more details.

A convenient way to describe the space of incomplete hyperbolic structures on $M=$ $\mathbf{S}^{3} \backslash K$ is to identify a hyperbolic structure with a $S L(2, \mathbb{C})$ structure. Then, the moduli

17 Notice, that our normalization of the Chern-Simons invariant agrees with [52,55, but differs from the normalization used in [36] by a factor of $2 \pi^{2}$. 
space of $S L(2, \mathbb{C})$ structures is described by the character variety which, in turn, is given by the zero locus of the A-polynomial [46],

$$
A(l, m)=0
$$

where $l$ and $m$ are the complex-valued 'eigenvalues' of the holonomies (2.10) around the longitude and the meridian of a knot. In these variables, the point in the moduli space representing the complete metric on $M$ is located at $(l, m)=(-1,1)$. The change of the volume and the Chern-Simons invariant is described, respectively, by the differential 1-forms (3.30) and (3.31) on the curve (5.5):

$$
\begin{aligned}
& d \operatorname{Vol}(M)=2(-\log |l| d(\arg m)+\log |m| d(\arg l)) \\
& d C S(M)=-\frac{1}{\pi^{2}}(\log |m| d \log |l|+(\arg l) d(\arg m))
\end{aligned}
$$

Therefore, if $K$ is a hyperbolic knot, the right-hand side of the volume conjecture (5.4) has a natural generalization. For a given knot $K$, we can consider a family of (incomplete) hyperbolic metrics on $M$ and regard $\operatorname{Vol}(M)$ and $C S(M)$ as functions on the curve $A(l, m)=0$ obtained by integrating (5.6), cf. fig. 7,

$$
\begin{aligned}
& \operatorname{Vol}(l, m)=\operatorname{Vol}(K)+2 \int(-\log |l| d(\arg m)+\log |m| d(\arg l)) \\
& C S(l, m)=C S(K)-\frac{1}{\pi^{2}} \int(\log |m| d \log |l|+(\arg l) d(\arg m))
\end{aligned}
$$

Here, $\operatorname{Vol}(K)$ and $C S(K)$ refer to the volume and the Chern-Simons invariant of the complete hyperbolic metric on the knot complement (more generally, the Gromov norm of $K)$.

In order to find a suitable generalization of the left-hand side of the volume conjecture (5.4), let us look at the path integral definition of the colored Jones polynomial. In quantum field theory, the colored Jones polynomial evaluated at the $k$-th root of unity appears as the normalized 18 expectation value of a Wilson line in the $S U(2)$ Chern-Simons theory [3],

$$
J_{N}\left(K, e^{2 \pi i / k}\right)=\left\langle\operatorname{Tr}_{R_{j}} \mathrm{P} \exp \oint_{K} A\right\rangle
$$

18 Here, the word "normalized" refers to a factor of $Z_{S U(2)}\left(\mathbf{S}^{3}\right)$ in the denominator of the path integral. In the following section we will introduce another version of the colored Jones polynomial, often used in the mathematical literature, where $J_{N}(K, q)$ is further normalized relative to $J_{N}($ unknot, $q)$. 
where $k$ stands for the (renormalized) value of the level, and $R_{j}$ is an irreducible spin- $j$ representation of $S U(2)$ of dimension $N=2 j+1$. Therefore, the left-hand side of the volume conjecture (5.4) can be interpreted as a classical limit of the Chern-Simons-Witten invariant (5.8), such that

$$
k \rightarrow \infty \quad, \quad N \rightarrow \infty \quad, \quad \frac{N}{k}=1
$$

In physics, Wilson lines represent trajectories of massive point-like particles. Interacting with gravity such particles produce conical defects in the geometry of space-time, so that the deficit angle is proportional to the mass of the particle [13]. These heuristic arguments suggest that, in the present context, a Wilson line (5.8) should be associated with a conical singularity along the knot $K$, with a deficit angle $\sim N / k$. On the other hand, deformations of the hyperbolic structure on $M$ with a conical singularity along the knot $K$ are parametrized by the curve (5.5), with $|m|=1$. Therefore, one might expect

$$
\log (m) \sim 2 \pi i\left(1-\frac{N}{k}\right)
$$

These considerations suggest that the proper modification of the left-hand side of the volume conjecture (5.4), that includes deformations of the hyperbolic structure on $M$, should be obtained by replacing (5.9) with a more general double-scaling limit,

$$
k \rightarrow \infty \quad, \quad N \rightarrow \infty \quad, \quad a \equiv \frac{N}{k}=\text { fixed }
$$

The ratio, $a=N / k$, which is kept fixed in this limit, does not need to be a rational number. In fact, the relation (5.10) suggests that, in order to compare with the volume and the Chern-Simons invariant computed from the A-polynomial, the parameter $a$ must be treated as a continuous complex variable. Moreover, rational values of $a$ (except for $a=1)$ are special, in a sense that the asymptotic behavior of the colored Jones polynomial "jumps" in such cases. Again, this suggests to consider generic values of $a$. Since the colored Jones polynomial is defined for all values of $q$, not only for the roots of unity, a natural way to realize this is to keep $N$ integer, and take $k=N / a$ to be non-integer 19 . Then, by analogy with (5.4), we expect the following conjecture to be true:

19 Alternatively, one could consider an analytic continuation of $J_{N}(K, q)$ to non-integer values of the color, $N$. Even though eventually this possibility may play an important róle in the connection with the $S L(2, \mathbb{C})$ Chern-Simons theory, it seems less obvious at present. See however [83], which may be relevant here. I wish to thank D. Thurston for pointing out this reference and for helpful discussions on related topics. 
The Generalized Volume Conjecture: In the limit (5.11), the $N$-colred Jones polynomial has the following asymptotic behavior:

$$
\lim _{N, k \rightarrow \infty} \frac{\log J_{N}\left(K, e^{2 \pi i / k}\right)}{k}=\frac{1}{2 \pi}\left(\operatorname{Vol}(l, m)+i 2 \pi^{2} C S(l, m)\right)
$$

where $\operatorname{Vol}(l, m)$ and $C S(l, m)$ are the functions (5.X) on the zero locus of the A-polynomial, evaluated at the point

$$
m=-\exp (i \pi a)
$$

This identification of the parameters agrees with the expected relation (5.10), where we fixed the exact numerical factor by considering specific examples, see below.

Note, that the generalized volume conjecture (5.12) presents a 1-parameter family of relations, which include the well-known volume conjecture (5.4) as a special case, $m=1$. Below, we demonstrate that (5.12) is true for the figure-eight knot.

\subsection{Proof of the Generalized Volume Conjecture for the Figure-Eight Knot $\mathbf{4}_{1}$}

The $N$-colored Jones polynomial of the figure-eight knot has the following form, see e.g. 84 :

$$
J_{N}\left(\mathbf{4}_{1}, q\right)=\sum_{i=0}^{N-1} \prod_{j=1}^{i}\left(q^{(N+j) / 2}-q^{-(N+j) / 2}\right)\left(q^{(N-j) / 2}-q^{-(N-j) / 2}\right)
$$

Notice, that at the $N$-th root of unity, $q=\exp (2 \pi i / N)$, it is indeed equal to the Kashaev's invariant of the figure-eight knot [34]:

$$
\left\langle\mathbf{4}_{1}\right\rangle_{N}=\sum_{i=0}^{N-1} \prod_{j=1}^{i}\left(1-q^{j}\right)\left(1-\bar{q}^{j}\right)
$$

In the limit (5.11), the asymptotic behavior of the colored Jones polynomial (5.14) can be obtained using the saddle-point approximation. The result has the following form, cf. 85]:

$$
\lim _{k, N \rightarrow \infty} \frac{\log J_{N}\left(\mathbf{4}_{1}, e^{2 \pi i / k}\right)}{k}=\frac{1}{\pi} V(a)
$$

where the function

$$
V(a)=\Lambda(a \pi+\theta(a) / 2)-\Lambda(a \pi-\theta(a) / 2)
$$

is defined via $\theta(a)=\arccos (\cos (2 \pi a)-1 / 2)$ and the Lobachevsky function,

$$
\Lambda(z)=-\int_{0}^{z} \log |2 \sin x| d x=\frac{1}{2} \sum_{n=1}^{\infty} \frac{\sin (2 n z)}{n^{2}}
$$

The graph of $V(a)$ is shown on the figure below: 


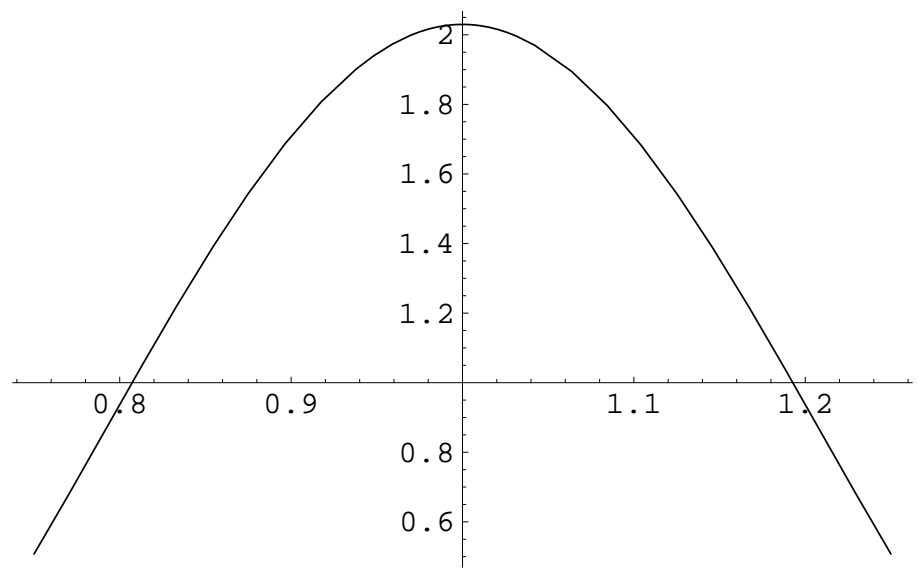

Fig. 9: The graph of the volume function $V(a)$ near $a=1$.

Notice, that at the special value $a=1$ corresponding to the complete hyperbolic structure on the knot complement, we have

$$
2 \pi \lim _{N \rightarrow \infty} \frac{\log J_{N}\left(\mathbf{4}_{1}, e^{2 \pi i / N}\right)}{N}=2(\Lambda(7 \pi / 6)-\Lambda(5 \pi / 6))
$$

Using the $\pi$-periodicity of the Lobachevsky function and the identity

$$
\Lambda(n z)=n \sum_{j(\bmod n)} \Lambda\left(z+\frac{\pi j}{n}\right)
$$

one can check that (5.18) is indeed equal to the volume of the figure-eight knot complement,

$$
\operatorname{Vol}\left(\mathbf{4}_{1}\right)=6 \Lambda(\pi / 3) \approx 2.0298832 \ldots
$$

Now, let us study the limit (5.15) as a function of the parameter $a$, near the point $a=1$. For reasons that will become clear in a moment, it is convenient to introduce a new parameter $y$, such that

$$
a=1+\frac{y}{\pi}
$$

According to the relation (5.13), we have

$$
m=\exp (i y)
$$

It is clear that $y$ is a good expansion parameter near $m=1$ (i.e. near $y=0$ ).

Since we already know that for $y=0$ the volume conjecture is true, we need to compare only the $y$-dependent terms on both sides in (5.12), say, by differentiating with respect to 
$y$. On the left-hand side, this leads to a derivative of the function (5.16), representing the limiting behavior of the colored Jones polynomial. Explicitly, we find

$$
\begin{aligned}
\frac{d V}{d y}= & \left(1-\frac{\sin (2 y)}{\sqrt{1-(\cos (2 y)-1 / 2)^{2}}}\right) \log \left|2 \sin \left(y-\frac{1}{2} \arccos (\cos (2 y)-1 / 2)\right)\right|- \\
& -\left(1+\frac{\sin (2 y)}{\sqrt{1-(\cos (2 y)-1 / 2)^{2}}}\right) \log \left|2 \sin \left(y+\frac{1}{2} \arccos (\cos (2 y)-1 / 2)\right)\right|
\end{aligned}
$$

It is easy to see that this is an odd function of $y$, which has the following power series expansion,

$$
\frac{d V}{d y}=-2 \sqrt{3} y+\frac{8}{\sqrt{3}} y^{3}-\frac{184}{15 \sqrt{3}} y^{5}+\frac{1424}{45 \sqrt{3}} y^{7}-\frac{86248}{945 \sqrt{3}} y^{9}+\ldots
$$

The graph of the function $d V / d y$ is shown on the figure below:

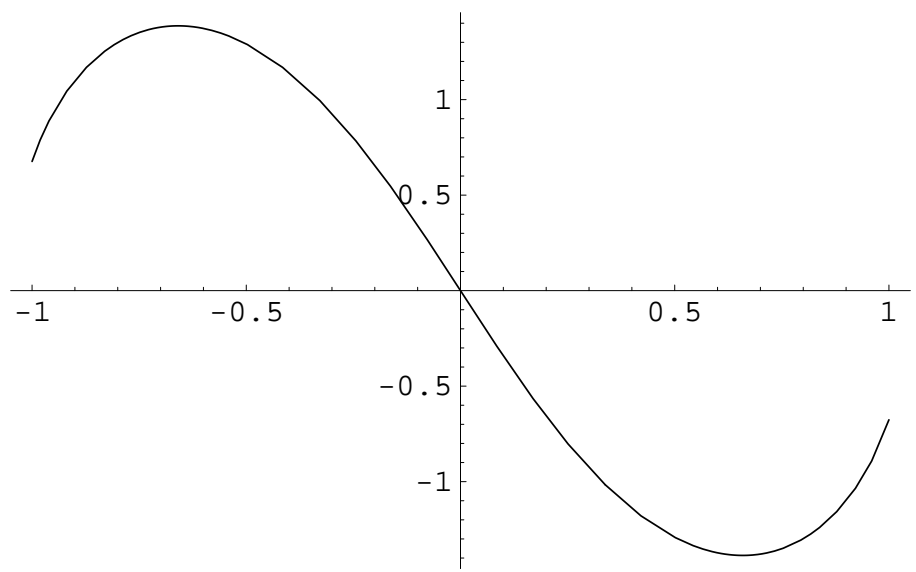

Fig. 10: The graph of the function $d V / d y$.

Now, let us look at the right-hand side of the proposed relation (5.12). It involves the volume function, $\operatorname{Vol}(l, m)$, and the Chern-Simons function, $C S(l, m)$, defined by the integrals (5.7) on the zero-locus of the A-polynomial. For the figure-eight knot, the Apolynomial has the following form 46]:

$$
A(l, m)=-2+m^{4}+m^{-4}-m^{2}-m^{-2}-l-l^{-1}
$$

For simplicity, let us take $y$ to be real. Then, using the fact that $C S\left(\mathbf{4}_{1}\right)=0$, we can write the integrals (5.7) in the following simple form,

$$
\operatorname{Vol}(m)=\operatorname{Vol}\left(\mathbf{4}_{1}\right)-2 \int_{0}^{y} \log |l| d(\arg m)
$$


and

$$
C S(m)=-\frac{1}{\pi^{2}} \int_{0}^{y}(\arg l) d(\arg m)
$$

where $\log |l|$ and $\arg l$ should be determined from the equation $A\left(l, e^{i y}\right)=0$. Since the A-polynomial (5.24) is quadratic in $l$, it is easy to solve this equation. One finds

$$
l=\cos (4 y)-\cos (2 y)-1 \pm \sqrt{(\cos (4 y)-\cos (2 y)-1)^{2}-1}
$$

It is the second root (with the "-" sign) that corresponds to the geometric branch of the moduli space of flat $S L(2, \mathbb{C})$ connections. Choosing this root, we find that $l$ is real for real values of $y$ (sufficiently close to $y=1$ ). Hence, the Chern-Simons integral (5.26) is identically zero. This agrees with the fact that the function $V$, that describes the limiting behavior of the colored Jones polynomial in (5.15) - (5.16) is also real. Therefore, the imaginary part of the proposed relation (5.12) is true — both sides vanish identically and we only need to verify the real part.

Fortunately, we do not need to evaluate the integral (5.25) explicitly in order to check the real part of the generalized volume conjecture (5.12). Since we are interested only in $y$-dependent terms, we only have to compare $(5.22)$ with the derivative of the volume function (5.25),

$$
-\log |l|=-\log \left[\cos (4 y)-\cos (2 y)-1-\sqrt{(\cos (4 y)-\cos (2 y)-1)^{2}-1}\right]
$$

It is amusing to verify that the functions (5.22) and (5.28) are indeed identical.

\subsection{A Relation Between The Colored Jones Polynomial And The A-Polynomial}

A generalization of the volume conjecture discussed above could be interpreted as a purely algebraic relation between the A-polynomial and the colored Jones polynomial. Specifically, given a colored Jones polynomial $J_{N}(K, q)$ of a knot $K$ one can consider the limit (5.11),

$$
k \rightarrow \infty \quad, \quad N \rightarrow \infty \quad, \quad a \equiv \frac{N}{k} \quad a \in \mathbb{C} \backslash \mathbb{Q}
$$

and define a function $l(a)$, such that $l(1)=-1$ and

$$
\log l=-\frac{d}{d a} \lim _{\substack{N, k \rightarrow \infty \\ N / k=a}} \frac{\log J_{N}\left(K, e^{2 \pi i / k}\right)}{k}
$$

From the definition, it might seem that $l(a)$ is a rather complicated function of $a$. However, the generalized volume conjecture (5.12) implies that $l$ and $m=-e^{i a}$ satisfy a simple 
polynomial relation (5.5) with integer coefficients. Namely, they belong to the zero locus of the A-polynomial.

\section{Remarks:}

1) Among other things, the generalized volume conjecture implies that the A-polynomial is an invariant of knots not stronger than the colored Jones polynomial (regarded as a function of the color as well). This seems to be consistent with the existing observations. For example, both the A-polynomial and the colored Jones polynomial can not distinguish mutants, etc.

2) Given that the colored Jones polynomial and the A-polynomial have a very different nature, it might be useful to understand a relation between them better. For instance, the colored Jones polynomial can be defined by skein relations, whereas no such definition is known for the A-polynomial.

3) It would be interesting to understand a relation to the work [86, where a similar connection between the A-polynomial and the Jones polynomial was proposed from the the non-commutative point of view.

\section{Non-Trivial Flat Connections and the Melvin-Morton-Rozansky Conjecture}

The volume conjecture and its generalization discussed in the previous section imply that, for generic values of $a$, the asymptotic behavior of the colored Jones polynomial is dominated by a flat $S L(2, \mathbb{C})$ connection. This observation forms a very nice and complete picture once considered along with the Melvin-Morton-Rozansky conjecture regarding the asymptotic behavior of the colored Jones polynomial for integer values of $k$. Using path integral interpretation, in this section we will try to explain this general picture, which will allow us to formulate an analog of the Melvin-Morton-Rozansky conjecture for the contribution of a non-trivial flat connection.

\subsection{A Brief Review of the Melvin-Morton-Rozansky Conjecture}

Let $J_{N}(K, q)$ be the $N$-colored Jones polynomial of a knot $K$. We define a reduced Jones polynomial as

$$
V_{N}(K, q)=\frac{J_{N}(K, q)}{J_{N}(\text { unknot }, q)}, \quad V_{N} \in \mathbb{Z}\left[q, q^{-1}\right]
$$


where $q=\exp (2 \pi i / k)$ and

$$
J_{N}(\text { unknot }, q)=[N]=\frac{q^{N / 2}-q^{-N / 2}}{q^{1 / 2}-q^{-1 / 2}}
$$

is the $N$-colored Jones polynomial for the trivial knot.

Following [87, let us denote by $J_{N}^{(t r)}(K, q)$ (resp. $\left.V_{N}^{(t r)}(K, q)\right)$ the trivial connection contribution to the colored Jones polynomial. Consider the Melvin-Morton expansion of the colored Jones polynomial in powers of $N$ and $h=q-1$ [88,

$$
V_{N}^{(t r)}(K, q)=\sum_{m, n \geq 0} D_{m, n}(K) N^{2 m} h^{n}
$$

Here, the rational numbers $D_{m, n}(K)$ are Vassiliev invariants of order $n$ [4, 68], and we assume that both $N$ and $k$ are integer. The extension to non-rational values of $a=N / k$ is related to the generalized volume conjecture and will be discussed further below.

It was conjectured by Melvin and Morton [88] and later proved by Rozansky [29], and by Bar-Natan and Garoufalidis [89], that the coefficients $D_{m, n}(K)$ in the expansion (6.3) have the following properties, 20 ,

$$
\begin{aligned}
& D_{m, n}=0 \quad \text { for } \quad m>n / 2 \\
& \sum_{m \geq 0} D_{m, 2 m} a^{2 m}=\frac{1}{\nabla_{A}\left(K, e^{i \pi a}-e^{-i \pi a}\right)}
\end{aligned}
$$

where $\nabla_{A}(K, z)$ is the Alexander polynomial of the knot $K$, normalized such that $\nabla_{A}($ unknot, $z)=1$. We remind, that the Alexander polynomial can be defined by the skein relation,

$$
\nabla_{A}\left(L_{+}, z\right)-\nabla_{A}\left(L_{-}, z\right)=z \nabla_{A}\left(L_{0}, z\right)
$$

corresponding to the link diagrams shown on the figure below.

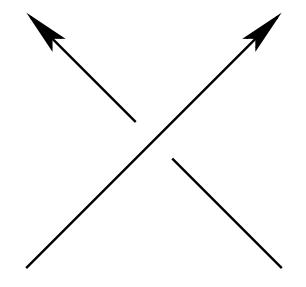

$L_{+}$

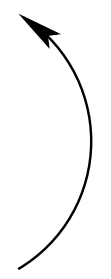

$L_{0}$

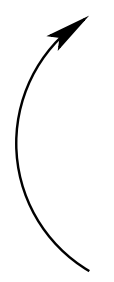

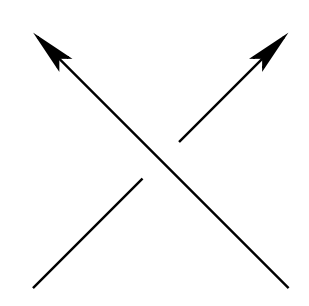

$L_{-}$

Fig. 11: Link diagrams connected by the skein relation.

20 To avoid cluttering, in what follows we suppress the dependence of $D_{m, n}(K)$ on the knot $K$. 
The bound on the powers of $N$ allows to rewrite (6.3) in the form

$$
V_{N}^{(t r)}(K, q)=\sum_{n \geq 0} h^{n} \sum_{m \geq 0} D_{m, n+2 m}(N h)^{2 m}
$$

which is reminiscent of the t' Hooft expansion in a $U(N)$ gauge theory. Let us rewrite this expansion in yet another form, replacing $(N h)$ with a new parameter

$$
z=q^{N / 2}-q^{-N / 2}=2 i \sin (\pi a)
$$

such that 90]:

$$
N h=2 \log \left(\sqrt{1+z^{2} / 4}+z / 2\right) \frac{h}{\log (1+h)}=z+\ldots
$$

Then, the Melvin-Morton expansion (6.6) of the colored Jones polynomial can be written in the form

$$
V_{N}^{(t r)}(K, q)=\sum_{n \geq 0} V^{(n)}(K, z) h^{n}=\sum_{n \geq 0} h^{n} \sum_{m \geq 0} d_{m}^{(n)} z^{2 m}
$$

In terms of the new variable $z$, the Melvin-Morton conjecture (6.4) turns into a statement about the first line in the expansion (6.8),

$$
V^{(0)}(K, z)=\frac{1}{\nabla_{A}(K, z)}
$$

which, among other things, implies that the coefficients $d_{m}^{(0)}$ are all integer numbers. Rozansky conjectured [90] and proved later 91], that all the coefficients $d_{m}^{(n)}$ are integer numbers and that the $n$-th line in the expansion (6.8) is a rational function of $z$ :

$$
V^{(n)}(K, z)=\frac{P^{(n)}(K, z)}{\nabla_{A}^{2 n+1}(K, z)} \quad, \quad P^{(n)}(K, z) \in \mathbb{Z}\left[z^{2}\right]
$$

In order to understand a connection between the Melvin-Morton-Rozansky and the volume conjectures, it is helpful to look at the path integral interpretation.

\subsection{Path Integral Interpretation}

As we already pointed out earlier, the colored Jones polynomial can be viewed as the expectation value of the Wilson loop observable (1.3) in the $S U(2)$ Chern-Simons theory [3],

$$
J_{N}\left(K, e^{\frac{2 \pi i}{k_{0}+2}}\right)=\left\langle W_{R_{j}}(K)\right\rangle=\frac{1}{Z_{S U(2)}\left(\mathbf{S}^{3}\right)} \int \mathcal{D} A W_{R_{j}}(K) e^{\frac{i k_{0}}{4 \pi} S_{C S}(A)}
$$


where $k_{0}$ is the tree-level value of the coupling constant, $R_{j}$ is an irreducible $N=(2 j+1)$ dimensional representation of $S U(2)$, and $S_{C S}(A)$ is the Chern-Simons functional of the $s u(2)$-values gauge connection $A$,

$$
S_{C S}=\operatorname{Tr} \int_{\mathbf{S}^{3}}\left(A \wedge d A+\frac{2}{3} A \wedge A \wedge A\right)
$$

The Wilson loop expectation value 6.11) is normalized by the Chern-Simons path integral on $\mathbf{S}^{3}$,

$$
Z_{S U(2)}\left(\mathbf{S}^{3}\right)=\sqrt{\frac{2}{k}} \sin \left(\frac{\pi}{k}\right)
$$

where $k=k_{0}+2$ is the renormalized value of the level.

In the semi-classical limit, $k \rightarrow \infty$, the path integral (6.11) can be represented as a sum over flat connections,

$$
Z_{S U(2)}\left(W_{R_{j}}, k\right)=\sum_{\alpha} Z_{S U(2)}^{(\alpha)}\left(W_{R_{j}}, k\right)
$$

where each term has the form of a perturbation series,

$$
Z_{S U(2)}^{(\alpha)}\left(W_{R_{j}}, k\right)=\exp \frac{i k}{4 \pi}\left(S_{C S}^{(\alpha)}+\sum_{n=1}^{\infty}(2 \pi / k)^{n} S_{n}^{(\alpha)}\right)
$$

Therefore, the leading contribution to the path integral (6.11) comes from the classical value of the Chern-Simons functional 6.12 ) evaluated on the flat connection $A^{(\alpha)}$. Then, the next, "1-loop" term, $S_{1}^{(\alpha)}$, has the form, see e.g. [3, 92,93,

$$
\exp \left(\frac{i}{2} S_{1}^{(\alpha)}\right)=\frac{\sqrt{T\left(A^{(\alpha)}\right)}}{\operatorname{Vol}\left(H_{\alpha}\right)}\left(\frac{4 \pi^{2}}{k}\right)^{\left(h^{0}\left(A^{(\alpha)}\right)-h^{1}\left(A^{(\alpha)}\right)\right) / 2} \exp \left(-\frac{i \pi}{4} N_{p h}\right)
$$

where $h^{k}\left(A^{(\alpha)}\right)$ is the dimension of the $k$-th cohomology of $M=\mathbf{S}^{3} \backslash K$ with coefficients twisted by $A^{(\alpha)}$, and $T\left(A^{(\alpha)}\right)$ is the $S U(2)$ Ray-Singer torsion of $M$ twisted by $A^{(\alpha)}$. The isotropy group, $H_{\alpha}$, is a subgroup of $S U(2)$ that commutes with the holonomies of the connection $A^{\alpha}$. Its tangent space, $T H_{\alpha}$, can be identified with the 0 -th cohomology space of $M$ twisted by $A^{(\alpha)}$. Therefore, we have

$$
h^{0}\left(A^{(\alpha)}\right)=\operatorname{dim}\left(H_{\alpha}\right)
$$

On the other hand, the elements of the 1-st twisted cohomology can be identified with infinitesimal deformations of the flat connection $A^{\alpha}$. However, since these deformations 
may be obstructed there is no simple formula for $h^{1}\left(A^{(\alpha)}\right)$, analogous to (6.17). Finally, $N_{p h}$ is given by

$$
N_{p h}=2 S F^{(\alpha)}+h^{0}\left(A^{(\alpha)}\right)+h^{1}\left(A^{(\alpha)}\right)+\left(1+b^{1}\right) \operatorname{dim} G
$$

where $S F^{(\alpha)}$ is a spectral flow of the self-adjoint operator $L=* D+D *$ restricted to the space of odd forms.

Now, following Rozansky [29], let us evaluate (6.16) in the case of a trivial connection.

\subsection{A Contribution of the Trivial Connection}

For the trivial connection we have $S_{C S}^{(t r)}=0$. Therefore, the leading contribution to the colored Jones polynomial comes from the 1-loop term. Since a reducible trivial connection with fixed boundary conditions on $M$ has no moduli, we have

$$
h^{0}=1 \quad, \quad h^{1}=0
$$

The isotropy group is $H_{\alpha}=U(1)$, and $\operatorname{Vol}\left(H_{\alpha}\right)=\sqrt{8} \pi$. Moreover, from the results of Milnor [94] and Turaev [95] it follows that in the present case the Ray-Singer torsion is related to the Alexander polynomial,

$$
\sqrt{T(a)}=\frac{2 \sin (\pi a)}{\nabla_{A}\left(K, e^{2 \pi i a}\right)}
$$

where $a=N / k$ is the $U(1)$ holonomy around the Wilson line $W_{R_{j}}(K)$. Therefore, substituting (6.19) and (6.20) into (6.16), we find that the leading contribution of the trivial connection is given by [29]:

$$
Z_{S U(2)}^{(t r)}\left(W_{R_{j}}, k\right) \simeq \sqrt{\frac{2}{k}} \frac{\sin (\pi a)}{\nabla_{A}\left(K, e^{2 \pi i a}\right)}
$$

Normalizing by (6.13), we find that in the limit $k \rightarrow \infty$ the contribution of the trivial connection to the colored Jones polynomial looks like

$$
J_{N}^{(t r)}\left(K, e^{2 \pi i / k}\right) \simeq \frac{k \sin (\pi a)}{\pi \nabla_{A}\left(K, e^{2 \pi i a}\right)}+\ldots
$$

This implies the following asymptotic behavior of the reduced Jones polynomial $V_{N}(K, q)$,

$$
V_{N}^{(t r)}\left(K, e^{2 \pi i / k}\right) \simeq \frac{1}{\nabla_{A}\left(K, e^{2 \pi i a}\right)}+\ldots
$$

which, in turn, implies the Melvin-Morton conjecture (6.4). 


\subsection{A Contribution of the "Hyperbolic" Flat Connection}

Now let us explain the relation to the volume conjecture and its generalization (5.12) discussed in the previous section. In the path integral interpretation, the exponential growth of the colored Jones polynomial means that its asymptotic behavior is dominated by the non-trivial $S L(2, \mathbb{C})$ flat connection, associated with the hyperbolic structure on the knot complement, $M$. We shall denote this connection by $A^{(\text {hyperb) }}$. Comparing (5.12) with (6.15), we conclude that the Chern-Simons action for this complex-valued connection is given by

$$
S_{C S}\left(A^{(h y p e r b)}\right)=-2 i\left(\operatorname{Vol}(m)+i 2 \pi^{2} C S(m)\right)
$$

For hyperbolic knots, the imaginary part of this expression does not vanish and, hence, leads to the exponential growth of $J_{N}(K, q)$ in the limit (5.11). Notice, that in order to see this exponential growth it is crucial to allow the parameter $a=N / k$ to take generic (non-rational) values, which is more natural in the $S L(2, \mathbb{C})$ Chern-Simons theory, rather than in the $S U(2)$ theory, $c f$. Table 1.

This means that we have to find a suitable generalization of the Melvin-Morton conjecture (6.4) for non-rational values of $a$. Such a generalization can be found using path integral arguments, similar to the above. Indeed, let us look at the loop expansion (6.15) of the Chern-Simons-Witten invariant around the flat connection $A^{\text {(hyperb) }}$. The leading term in this expansion is given by the classical action (6.24). In the next, 1-loop term we have

$$
h^{0}\left(A^{(\text {hyperb })}\right)=0 \quad, \quad h^{1}\left(A^{(\text {hyperb })}\right)=0
$$

This follows from eq.(6.17) and the fact that the flat connection $A^{(\text {hyperb) }}$, associated with the hyperbolic structure on the knot complement, is irreducible and rigid, so that $\operatorname{dim}\left(H_{\text {hyperb }}\right)=0$. (In fact, the same relations describe the contribution of a non-trivial flat connection to the Reshetikhin-Turaev-Witten invariant for torus knots [29].) Therefore, from (6.15) we get,

$$
Z_{S U(2)}^{(\text {hyperb })}\left(W_{R_{j}}, k\right)=\sqrt{T\left(A^{(\text {hyperb })}\right)} e^{\frac{i k}{4 \pi}\left(S_{C S}^{(\text {hyperb })}+\sum_{n>1}(2 \pi / k)^{n} S_{n}^{(h y p e r b)}\right)}
$$

where we ignore a constant phase. In this expression, $T\left(A^{(\text {hyperb })}\right)$ denotes the $S L(2, \mathbb{C})$ Ray-Singer torsion of the knot complement twisted by $A^{\text {(hyperb) }}$. Normalizing by $Z_{S U(2)}\left(\mathbf{S}^{3}\right)$, we find the asymptotic behavior of the $N$-colored Jones polynomial,

$$
J_{N}(K, q)=\frac{\sqrt{k}}{\sqrt{2} \sin (\pi / k)} \sqrt{T} \exp \left(\frac{k}{2 \pi}\left(\operatorname{Vol}(m)+i 2 \pi^{2} C S(m)\right)+\frac{i}{2} \sum_{n=1}^{\infty}(2 \pi / k)^{n} S_{n+1}\right)
$$


It is convenient to write this expression in the logarithmic form:

$$
\log J_{N}(K, q)=\frac{k}{2 \pi}\left(\operatorname{Vol}(m)+i 2 \pi^{2} C S(m)\right)+\log \left(\frac{\sqrt{k T}}{\sqrt{2} \sin (\pi / k)}\right)+\frac{i}{2} \sum_{n=1}^{\infty}(2 \pi / k)^{n} S_{n+1}
$$

Notice, that this expansion is very similar to the perturbative expansion of the $S L(2, \mathbb{C})$ partition function, $c f$. (3.38) - (3.39). It would be very interesting to find a better understanding of this relation.

Now, by analogy with (6.1), let us define a properly normalized version of the colored Jones polynomial that would remain finite in the limit (5.11). In eq.(6.1) this was achieved by dividing by the colored Jones polynomial of the unknot, which automatically removed the linear $k$-dependence from (6.22), as well as the universal factor of $\sin (\pi a)$. By considering specific examples (see below), we find that the Ray-Singer torsion, $T\left(A^{(h y p e r b)}\right)$, does not have the universal factor $\sin (\pi a)$ in the present case. Moreover, as can be easily seen from (6.27), the polynomial growth of $J_{N}(K, q)$ comes from the normalization of the Chern-Simons partition function (6.26) by $Z_{S U(2)}\left(\mathbf{S}^{3}\right)$. Therefore, in the present case, it is natural to define the reduced Jones polynomial $\widetilde{V}_{N}(K, q)$ by restoring the original normalization in (6.26),

$$
\widetilde{V}_{N}(K, q)=J_{N}(K, q) \cdot Z_{S U(2)}\left(\mathbf{S}^{3}\right) \cdot \exp \left(-\frac{k}{2 \pi}\left(\operatorname{Vol}(m)+i 2 \pi^{2} C S(m)\right)\right)
$$

where we also explicitly eliminated the exponential growth using the volume and the ChernSimons functions introduced in (5.7). It follows from the loop expansion (6.26), that the resulting Jones polynomial has the following asymptotic behavior,

$$
\widetilde{V}_{N}(K, q)=\sqrt{T\left(A^{(\text {hyperb })}\right)} \cdot \exp \left(\frac{i}{2} \sum_{n=1}^{\infty}(2 \pi / k)^{n} S_{n+1}\right)
$$

In particular, this expression remains finite in the limit (5.11). Hence, as in the case of the trivial flat connection, we can consider the Melvin-Morton expansion of $\widetilde{V}_{N}(K, q)$,

$$
\widetilde{V}_{N}(K, q)=\sum_{m, n \geq 0} \widetilde{D}_{m, n} N^{m} h^{n}
$$

The perturbative expansion (6.15) of the Chern-Simons path integral implies that the coefficients $\widetilde{D}_{m, n}$ should vanish unless $m \leq n$. Moreover, the following analog of the Melvin-Morton conjecture should hold in the limit (5.11), with non-rational $a$, 


\section{The Generalized Melvin-Morton Conjecture:}

$$
\begin{aligned}
& \widetilde{D}_{m, n}=0 \quad \text { for } \quad m>n \\
& \sum_{m \geq 0} \widetilde{D}_{m, m} a^{m}=\sqrt{T\left(A^{(\text {hyper })}\right)}
\end{aligned}
$$

To get further insights into geometric information encoded in the power series $(6.29)$, following [90], let us write it in terms of the variable $z=q^{N / 2}-q^{-N / 2}=2 i \sin (\pi a)$,

$$
\widetilde{V}_{N}(K, q)=\sum_{n \geq 0} \widetilde{V}^{(n)}(K, z) h^{n}=\sum_{n \geq 0} h^{n} \sum_{m \geq 0} \widetilde{d}_{m}^{(n)} z^{m}
$$

In contrast to the case of the reducible connection, the numbers $\widetilde{d}_{m}^{(n)}$ do not appear to be integer. However, it is plausible that $\widetilde{d}_{m}^{(n)}$ take values in $\mathbb{Q}(\sqrt{-1})$, or in some other number field, which might be related to the arithmetic properties of the knot complement.

\section{Questions:}

1) What is the geometric interpretation of the numbers $\widetilde{D}_{m, n}$ and $\widetilde{d}_{m}^{(n)}$ ?

2) What is the relation, if any, between $\widetilde{d}_{m}^{(n)}$ and the coefficients $b_{m, n}$ in the perturbative expansion (3.39) of the $S L(2, \mathbb{C})$ partition function?

Example. The Figure-eight Knot

As we explained above, in order to verify the generalized version of the MelvinMorton conjecture (6.30), it is enough to check that the colored Jones polynomial has asymptotic expansion of the form (6.27). For the figure-eight knot we find the following asymptotic behavior of the colored Jones polynomial,

$$
\log J_{N}\left(\mathbf{4}_{1}, e^{2 \pi i / k}\right)=\frac{k}{2 \pi} \operatorname{Vol}\left(\mathbf{4}_{1}\right)+\frac{3}{2} \log k+\frac{i}{2} S_{1}(a)+\frac{i}{2} S_{2}(a)\left(\frac{2 \pi}{k}\right)+\ldots
$$

where the dominant linear term was identified earlier in (5.18), and the coefficient of the logarithmic term agrees with the expected cohomology (6.25) and with the general formula (6.27). The subleading terms, $S_{n}(a)$, contain the information about the coefficients $\widetilde{D}_{m, n}$ and $\widetilde{d}_{m}^{(n)}$. Numerically, we find

$$
\begin{aligned}
& \widetilde{d}_{0}^{(0)}=3.3755 \ldots \\
& \widetilde{d}_{0}^{(1)}=-i 0.301 \ldots \\
& \widetilde{d}_{0}^{(2)}=0.06 \ldots+i 0.151 \ldots
\end{aligned}
$$




\section{Acknowledgments}

It is a pleasure to thank D. Bar-Natan, R. Dijkgraaf, N. Dunfield, S. Garoufalidis, R. Gopakumar, G. Horowitz, D. Long, M. Mariño, S. Minwalla, H. Ooguri, F. RodriguezVillegas, L. Rozansky, C. Vafa, E. Witten, S.-T. Yau, and especially K. Krasnov, G. Moore, A. Strominger, and D. Thurston for valuable and stimulating discussions. This research was conducted during the period S.G. served as a Clay Mathematics Institute Long-Term Prize Fellow. This work is also supported in part by RFBR grant 01-01-00549 and RFBR grant for Young Scientists 02-01-06322. I would also like to thank the University of California at Santa Barbara, Stanford University, California Institute of Technology, and Rutgers University for kind hospitality while this work was in progress.

\section{Appendix A. The BTZ Black Hole}

The metric of the Lorentzian BTZ black hole is described by the line element 21 [96]:

$$
d s^{2}=-\left(r^{2}-M\right) d t^{2}+\left(r^{2}-M\right)^{-1} d r^{2}+r^{2} d \phi^{2}
$$

Analytic continuation leads to the Euclidean metric

$$
d s^{2}=\left(r^{2}-M\right) d t^{2}+\left(r^{2}-M\right)^{-1} d r^{2}+r^{2} d \phi^{2}
$$

This is a special case of a more general metric corresponding to a rotating BTZ black hole. Namely, a solution corresponding to a black hole with mass $M$ and angular momentum $J$ assumes the following form:

$$
\begin{aligned}
d s^{2} & =N^{2} d \tau^{2}+N^{-2} d r^{2}+r^{2}\left(d \phi^{2}+N^{\phi} d \tau\right)^{2} \\
N & =\sqrt{r^{2}-M-\frac{J^{2}}{4 r^{2}}}, \quad N^{\phi}=-\frac{J}{2 r^{2}} \\
r_{ \pm}^{2} & =\frac{M}{2}\left[1 \pm \sqrt{1+\left(\frac{J}{M}\right)^{2}}\right]
\end{aligned}
$$

This geometry can be represented as a quotient (2.8) of the hyperbolic 3-space,

$$
M=\mathbb{H}^{3} / \Gamma
$$

21 We remind that, in our notations, $\ell=1$. 
by a discrete group $\Gamma$. The explicit change of variables, which brings the Euclidean metric (A.3) to the standard hyperbolic metric (2.7) on $\mathbb{H}^{3}$, is given by

$$
\begin{aligned}
& x=\sqrt{\frac{r^{2}-r_{+}^{2}}{r^{2}-r_{-}^{2}}} \cdot \cos \left(r_{+} \tau+\left|r_{-}\right| \phi\right) \cdot e^{r_{+} \phi-\left|r_{-}\right| \tau} \\
& y=\sqrt{\frac{r^{2}-r_{+}^{2}}{r^{2}-r_{-}^{2}}} \cdot \sin \left(r_{+} \tau+\left|r_{-}\right| \phi\right) \cdot e^{r_{+} \phi-\left|r_{-}\right| \tau} \\
& z=\sqrt{\frac{r_{+}^{2}-r_{-}^{2}}{r^{2}-r_{-}^{2}}} \cdot e^{r_{+} \phi-\left|r_{-}\right| \tau}
\end{aligned}
$$

In order to identify the quotient group $\Gamma$, it is convenient to write $(x, y, z)$ in terms of the spherical coordianates,

$$
\begin{aligned}
& x=R \cos \theta \cos \chi \\
& y=R \sin \theta \cos \chi \\
& z=R \sin \chi
\end{aligned}
$$

In these variables, the black hole metric has the form

$$
d s^{2}=\frac{1}{\sin ^{2} \chi}\left(\frac{d R}{R}\right)^{2}+\cot ^{2} \chi d \theta^{2}+\frac{1}{\sin ^{2} \chi} d \chi^{2}
$$

and the identifications on $\mathbb{H}^{3}$ that generate the holonomy group $\Gamma$ are

$$
\begin{aligned}
& (a): \quad(R, \theta, \chi) \sim\left(R \cdot e^{2 \pi r_{+}}, \theta+2 \pi\left|r_{-}\right|, \chi\right) \\
& (b): \quad(R, \theta, \chi) \sim(R, \theta+2 \pi, \chi)
\end{aligned}
$$

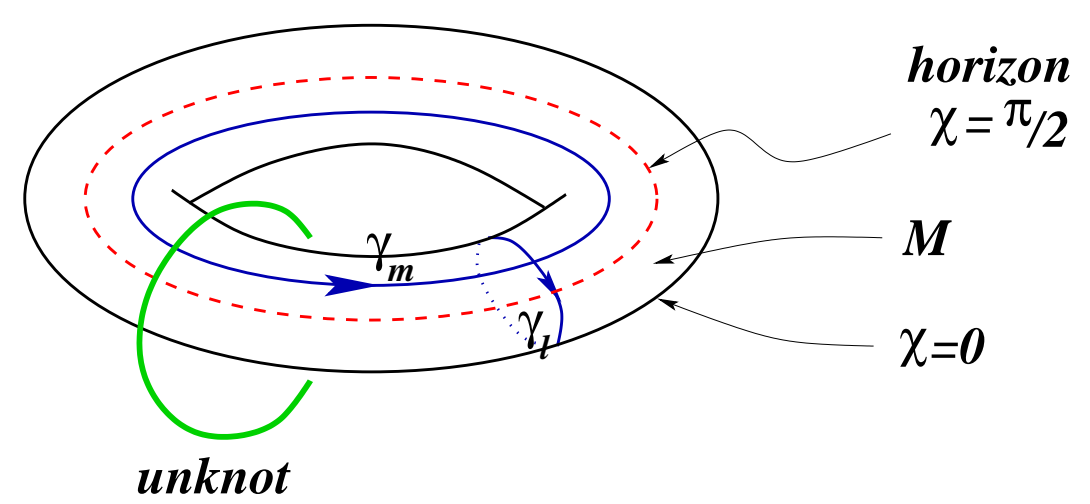

Fig. 12: The Euclidean BTZ black hole has a geometry of the solid torus, $M \cong$ $\mathbf{D}^{2} \times \mathbf{S}^{1}$. We can also view $M$ as a complement of the unknot (the trivial knot) in the 3 -sphere. 
Using (A.5) and (A.7), it is easy to see that the resulting geometry is a solid torus, $M \cong \mathbf{D}^{2} \times \mathbf{S}^{1}$, shown on fig. 12. The core of the torus (the circle $\chi=\pi / 2$ ) corresponds to the position of the "horizon". The solid torus $M$ can be also viewed as a complement of the unknot (the trivial knot) in the 3-sphere, $M=\mathbf{S}^{3} \backslash K$. The tubular neighborhood of the trivial knot $K$ is itself homeomorphic to a solid torus, $c f$. fig. 5. Thus, a union of these two solid tori joined along a common torus boundary gives back the 3-sphere.

Notice, that the longitude $\gamma_{l}$ of the unknot is contractible in $M$, whereas the meridian $\gamma_{m}$ is a non-trivial cycle in $M$. Therefore, it follows that the holonomy $l$ must be trivial, whereas the value of $m$ can be arbitrary. Hence, we conclude that the A-polynomial of the unknot has the following simple form, $c f$. (2.16),

$$
A_{\text {unknot }}(l, m)=l-1
$$

It turns out that this result has a nice physical interpretation. Namely, the vanishing of the polynomial (A.8) can be viewed as a "mass shell" condition for the so-called off-shell BTZ black hole, which we are going to describe next.

The geometry of the off-shell BTZ black hole can be obtained from (A.7) by replacing the second identification with a more general condition [49,50,51,28]:

$$
\left(b^{\prime}\right): \quad(R, \theta, \chi) \sim\left(e^{\Sigma} \cdot R, \theta+\Theta, \chi\right)
$$

The resulting identifications (A.7) - (A.9) can be represented by the elements of the $S L(2, \mathbb{C})$ group,

$$
\rho(a)=\left(\begin{array}{cc}
e^{\pi\left(r_{+}+i\left|r_{-}\right|\right)} & 0 \\
0 & e^{-\pi\left(r_{+}+i\left|r_{-}\right|\right)}
\end{array}\right) \quad, \quad \rho\left(b^{\prime}\right)=\left(\begin{array}{cc}
e^{(\Sigma+i \Theta) / 2} & 0 \\
0 & e^{-(\Sigma+i \Theta) / 2}
\end{array}\right)
$$

acting on the hyperbolic 3-space as in (2.9). These two elements generate the holonomy group $\Gamma \subset S L(2, \mathbb{C})$, such that the quotient space $M=\mathbb{H}^{3} / \Gamma$ is a solid torus with a conical singularity at the "horizon", which resembles the space-time geometry around the spinning particle in $2+1$ dimensions [13]. In this more general family of solutions, the usual BTZ black hole metric without a conical singularity is reproduced when

$$
\Sigma=0 \quad, \quad \Theta=2 \pi
$$

Following the standard terminology, we refer to this condition as the "mass shell" condition. 
In what follows, our goal will be to construct a flat $S L(2, \mathbb{C})$ connection corresponding to the more general class of BTZ black home geometries and, in particular, to demonstrate that the mass shell condition (A.11) is equivalent to the vanishing of the A-polynomial (A.8). For this, we need to evaluate the holonomies of the gauge connection,

$$
\rho(\gamma)=P \exp \oint_{\gamma} \mathcal{A}
$$

along the longitude, $\gamma_{l}$, and the meridian, $\gamma_{m}$. One can choose to parameterize these cycles by a real variable $s \in[0,1]$, such that

$$
\begin{aligned}
\gamma_{m}: & s \mapsto\left(R_{0} e^{2 \pi r_{+} s}, \theta_{0}+2 \pi\left|r_{-}\right| s, \chi_{0}\right) \\
\gamma_{l}: & s \mapsto\left(R_{0} e^{s \Sigma}, \theta_{0}+s \Theta, \chi_{0}\right)
\end{aligned}
$$

Notice, that due to the identifications (A.7) - (A.9), the curves $\gamma_{m}$ and $\gamma_{l}$ are manifestly closed.

In order to evaluate the holonomies (A.12), we also need to construct the complex valued gauge connection, $\mathcal{A}^{a}=w^{a}+i e^{a}$, from the components of the vielbein and the spin connection in the Euclidean BTZ black hole geometry. The latter can be taken, for example, in the following form [50,51]:

$$
\begin{aligned}
e^{1} & =\frac{1}{\sin \chi} \frac{d R}{R} & w^{1} & =-\frac{1}{\sin \chi} d \theta \\
e^{2} & =\frac{1}{\sin \chi} d \chi & w^{2} & =0 \\
e^{3} & =\cot \chi \cdot d \theta & w^{3} & =\cot \chi \cdot \frac{d R}{R}
\end{aligned}
$$

It is easy to verify that the corresponding $S L(2, \mathbb{C})$ gauge connection is indeed flat. However, as we shall see in a moment, this choice of the vielbein and the spin connection leads to a singular connection $22, \mathcal{A}$. Indeed, even though (A.14) defines a smooth metric (A.6), it corresponds to a singular gauge field, $\mathcal{A}^{1}=w^{1}+i e^{1}$, which is not well defined near the center of the solid torus, $\chi=\pi / 2$, where the angular variable $\theta$ is ill-defined and $w^{1} \approx-d \theta$.

Another way to see that (A.14) does not correspond to a smooth $S L(2, \mathbb{C})$ connection over $M$ is to evaluate the holonomies (A.12) around the 1-cycles (A.13). The resulting holonomies turn out to be in the same conjugacy class as the generating elements (A.10)

22 I am endebted to G. Moore and A. Strominger for very helpful comments and suggestions on these points. 
of the group $\Gamma$. Therefore, comparing (A.10) with (2.10), we conclude that in the present case the holonomies $l$ and $m$ are given by

$$
l=e^{(\Sigma+i \Theta) / 2} \quad, \quad m=e^{\pi\left(r_{+}+i\left|r_{-}\right|\right)}
$$

In particular, we find that, with the choice (A.14), the "mass shell" condition (A.11) looks like

$$
l+1=0
$$

and has the 'wrong' sign compared to (A.8). This result does not agree with the fact that the 1-cycle $\gamma_{l}$ becomes contractible in the on-shell BTZ black hole geometry and, therefore, the corresponding holonomy should be trivial. Finally, let us remark that this problem can not be fixed by applying a gauge transformation to (A.14) since any (non-singular) gauge transformation can not change the holonomy.

Therefore, we need to construct a flat $S L(2, \mathbb{C})$ gauge connection that would be nonsingular everywhere inside $M$. It is convenient to introduce a new set of coordinates:

$$
\begin{aligned}
& \varrho=\log R \\
& \xi=f(\chi) \cos \theta \\
& \eta=f(\chi) \sin \theta
\end{aligned}
$$

where the function,

$$
f(\chi)=\frac{\cos \chi}{1+\sin \chi}
$$

is chosen such that the metric induced in the $(\xi, \eta)$-plane is conformal to the usual Euclidean metric, $d s^{2}=d \xi^{2}+d \eta^{2}$. Specifically, rewriting the BTZ black hole metric (A.6) in the coordinates (A.17), we find

$$
d s^{2}=\left(\frac{1+f^{2}}{1-f^{2}}\right)^{2}\left[d \varrho^{2}+\frac{4}{\left(1+f^{2}\right)^{2}}\left(d \xi^{2}+d \eta^{2}\right)\right]
$$

The corresponding components of the vielbein and the spin connection can be written as

$$
\begin{aligned}
e^{1} & =\frac{1+f^{2}}{1-f^{2}} d \varrho & w^{1} & =\xi e^{3}-\eta e^{2} \\
e^{2} & =\frac{2}{1-f^{2}} d \xi & w^{2} & =\frac{2 \eta}{1+f^{2}} e^{1} \\
e^{3} & =\frac{2}{1-f^{2}} d \eta & w^{3} & =-\frac{2 \xi}{1+f^{2}} e^{1}
\end{aligned}
$$


Combining these together we find the explicit expression for the components of the flat $S L(2, \mathbb{C})$ gauge connection, $\mathcal{A}^{a}=w^{a}+i e^{a}$,

$$
\begin{aligned}
\mathcal{A}^{1} & =\frac{2 \xi}{1-f^{2}} d \eta-\frac{2 \eta}{1-f^{2}} d \xi+i \frac{1+f^{2}}{1-f^{2}} d \varrho \\
\mathcal{A}^{2} & =\frac{2 \eta}{1-f^{2}} d \varrho+i \frac{2}{1-f^{2}} d \xi \\
\mathcal{A}^{3} & =-\frac{2 \xi}{1-f^{2}} d \varrho+i \frac{2}{1-f^{2}} d \eta
\end{aligned}
$$

which is non-singular everywhere in the interior of the solid torus, $M=\mathbf{S}^{1} \times \mathbf{D}^{2}$.

Now let us evaluate the $S L(2, \mathbb{C}$ ) holonomies (A.12). For simplicity, let us consider a non-rotating BTZ black hole, with $J=0$. Then, the second identification in (A.7) is trivially realized in the coordinates (A.17), whereas the first one reads:

$$
(a): \quad(\varrho, \xi, \eta) \sim\left(\varrho+2 \pi r_{+}, \xi, \eta\right)
$$

Therefore, we can choose the cycles $\gamma_{l}$ and $\gamma_{m}$ to be parametrized in the following way, $c f$. (A.13),

$$
\begin{aligned}
\gamma_{m}: & s \mapsto\left(\varrho_{0}+2 \pi r_{+} s, \xi=0, \eta=0\right) \\
\gamma_{l}: \quad s & \mapsto\left(\varrho_{0}, \xi=\xi_{0} \cos 2 \pi s, \eta=\xi_{0} \sin 2 \pi s\right)
\end{aligned}
$$

Since only the $\mathcal{A}^{1}$-component of the gauge connection is non-trivial along the curve $\gamma_{m}$, we can easily find the corresponding holonomy,

$$
\rho\left(\gamma_{m}\right)=\left(\begin{array}{cc}
e^{\pi r_{+}} & 0 \\
0 & e^{-\pi r_{+}}
\end{array}\right)
$$

Comparing this result with (A.15), we find that the holonomy of the gauge connection (A.21) around $\gamma_{m}$ is the same as in the previous calculation, based on the singular gauge connection (A.14). On the other hand, since the connection (A.21) is flat and non-singular everywhere in the interior of the solid torus, the holonomy around a contractible cycle $\gamma_{l}$ is guaranteed to vanish automatically,

$$
l=1
$$

However, since many components of the gauge connection (A.21) do not vanish along the curve $\gamma_{l}$, to verify this directly would require some work. Perhaps the best way to approach this problem would be to find a gauge transformation, which removes non-commuting components of the gauge connection, and allows to write the holonomy (A.12) in a simple form. 


\section{Appendix B. Quantization for Torus Knots}

One simple way of classifying knots (in $\mathbb{R}^{3}$ ) is to associate to every knot $K$ a nonnegative number, $g(K)$, called the genus of the knot. Indeed, every knot can be embedded in some Riemann surface of genus $g$, and, as the name suggests, $g(K)$ is the minimal value of genus for which this can be done (without self-crossing or breaking the knot). For example, the only knot with $g=0$ is the unknotted circle. The next simplest case corresponds to knots of genus one, the so-called torus knots.

Since torus knots are special in a number of ways, we can't really call them generic representatives. In particular, since torus knots are not hyperbolic, most of the motivation discussed in the introduction does not apply here. Nevertheless, torus knots provide a simple toy model, which includes all the ingredients that one would find in a more complicated non-linear quantum system, say, corresponding to a figure-eight knot. Therefore, in order to emphasize the general ideas, here we will ignore the subtleties, slightly oversimplifying our discussion.
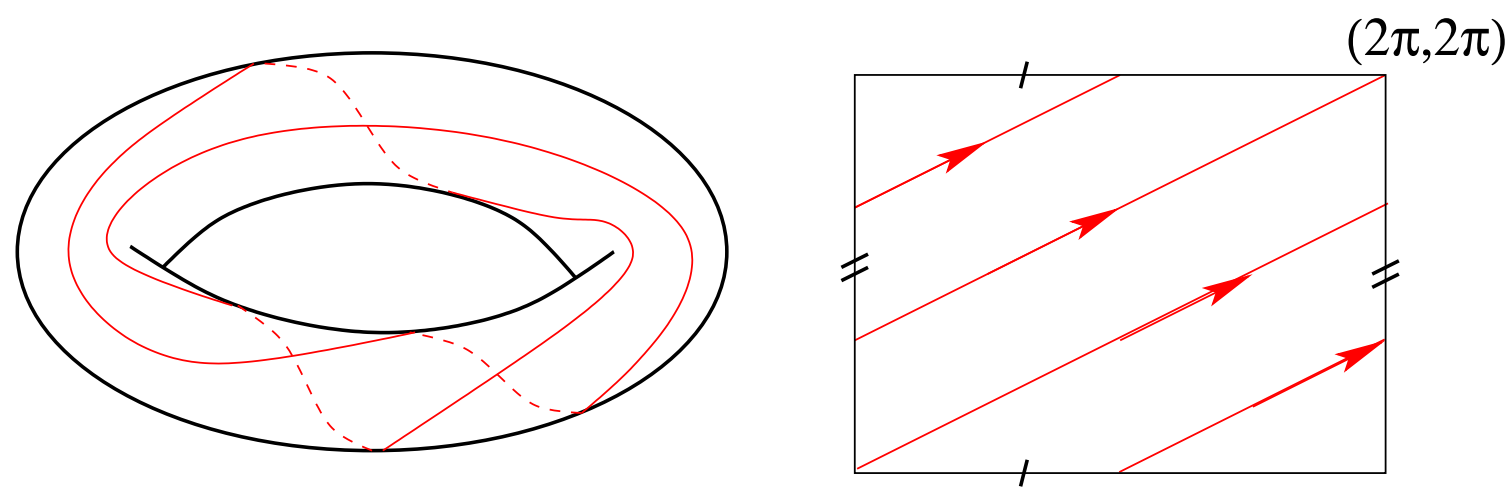

$(0,0)$

Fig. 13: Trefoil knot on a torus.

By definition, torus knots are knots which can be drawn on the surface of a torus. They are labeled by an unordered pair of relatively prime numbers $(r, s)$, which refers to the number of times the image of the knot winds basic cycles on the torus. For example, the trefoil knot is a torus knot of type $(2,3)$, see fig. 13 .

The A-polynomial of a non-trivial $(r, s)$-torus knot has a very simple form [46]:

$$
A(l, m)=l m^{r s}+1
$$


The zero locus of this polynomial defines a character variety $L$, linearly embedded into $\mathcal{P}=\mathbb{C}^{*} \times \mathbb{C}^{*}$. In terms of the $(u, v)$-variables $(3.4)$, the variety $L$ can be defined by the following two equations:

$$
\begin{aligned}
& \operatorname{Re}(u)+r s \cdot \operatorname{Re}(v)=0 \\
& \operatorname{Im}(u)+r s \cdot \operatorname{Im}(v)-\pi=0
\end{aligned}
$$

Our goal is to quantize a Hamiltonian system associated with $L \hookrightarrow \mathcal{P}$ and, in particular, to find the quantum wave function $Z(M)$ supported on $L$. The result can be interpreted as a partition function in the $S L(2, \mathbb{C})$ Chern-Simons theory on a complement $M=\mathbf{S}^{3} \backslash K$ of the torus knot $K$. Notice, that $M$ is not a hyperbolic 3-manifold. Therefore, as suggested by the asymptotic expression (3.34), it is natural to consider a special case 23 corresponding to $\sigma=0$.

In this case, the canonical commutation relations that follow from the Chern-Simons action (1.1) look like:

$$
\begin{aligned}
& {[\operatorname{Re}(v), \operatorname{Re}(u)]=[\operatorname{Im}(u), \operatorname{Im}(v)]=\frac{2 \pi i}{k}} \\
& {[\operatorname{Re}(u), \operatorname{Im}(v)]=-[\operatorname{Re}(v), \operatorname{Im}(u)]=0}
\end{aligned}
$$

and the Liouville 1-form (3.26) is given by

$$
\theta=\frac{k}{\pi}(\operatorname{Re}(v) d \operatorname{Re}(u)+\operatorname{Im}(u) d \operatorname{Im}(v))
$$

It can be written in the canonical form (3.16) provided that we define the coordinates $q_{i}$ and the conjugate momenta $p_{j}$ as

$$
\left(\begin{array}{l}
p_{1} \\
p_{2}
\end{array}\right)=\left(\begin{array}{c}
\operatorname{Re}(v) \\
\operatorname{Im}(u)
\end{array}\right) \quad \text { and } \quad\left(\begin{array}{c}
q_{1} \\
q_{2}
\end{array}\right)=\left(\begin{array}{c}
\operatorname{Re}(u) \\
\operatorname{Im}(v)
\end{array}\right)
$$

Here we omit the overall factor $k / 4 \pi$, which can be treated as the inverse Planck constant. In these variables, the Lagrangian submanifold $L$ can be written as:

$$
\begin{aligned}
& q_{1}+r s \cdot p_{1}=0 \\
& r s \cdot q_{2}+p_{2}-\pi=0
\end{aligned}
$$

Notice, that the Hamiltonian system splits into two non-interacting subsystems described by the canonical variables $\left(q_{1}, p_{1}\right)$ and $\left(q_{2}, p_{2}\right)$, respectively. The phase space of

23 In another special case, $k=0$ and $\sigma \neq 0$, we find that the action integral $S$ is identically zero. 
the first system is non-compact, whereas in the second system both the coordinate and the momentum are compact. For now, let us ignore the compactness of $q_{2}$ and $p_{2}$. Then, in the coordinate representation the action integral reads:

$$
\begin{aligned}
S & =\frac{k}{\pi} \int p_{1} d q_{1}+p_{2} d q_{2}= \\
& =-\frac{k}{\pi}\left(\frac{1}{2 r s} q_{1}^{2}+\frac{r s}{2} q_{2}^{2}-\pi q_{2}\right)
\end{aligned}
$$

If $\psi$ is a constant half-density on $L$, then the transformation rule for half-densities implies that the Reidemeister-Ray-Singer torsion, $T$, is also constant (independent on $q_{i}$ ) in this class of examples. Therefore, in the semi-classical limit the partition function of the $S L(2, \mathbb{C})$ Chern-Simons theory on the complement of a torus knot looks like:

$$
Z(M) \sim \exp \left(-\frac{i k}{2 \pi r s} q_{1}^{2}-\frac{i k r s}{2 \pi} q_{2}^{2}+i k q_{2}\right)+\ldots
$$

This result gives a prediction for the Chern-Simons invariant of the torus knot complement $M$ (as a function of the holonomies $l$ and $m$ ). Using (B.2) and (B.5), we can write it as:

$$
C S(M)=\frac{1}{2 \pi^{2} r s}\left(\log ^{2}|l|+\arg ^{2}(l)-\pi^{2}\right)
$$

Here, we slightly oversimplified our discussion assuming that the phase spaces of both dynamical systems are non-compact. A nice way to incorporate the compactness of $q_{2}$ and $p_{2}$ is to notice that they parameterize a torus 24 , which can be viewed as a phase space of the $S U(2)$ Chern-Simons theory on a 3 -manifold $M$ with boundary $\Sigma$, see (2.13). Hence, the exact partition function of the $S L(2, \mathbb{C})$ Chern-Simons theory on $M$ can be written as a product,

$$
Z_{S L(2, \mathbf{C})}(M)=Z_{S U(2)}(M) \times \exp \left(-\frac{i k}{2 \pi r s} q_{1}^{2}+\frac{i \pi}{4} \operatorname{sign}(r s)\right)
$$

where $Z_{S U(2)}(M)$ is the partition function of the $S U(2)$ Chern-Simons theory (see e.g. [43, 61, 97, 98, 99] ), and the rest represents the wave function in the first system, with noncompact phase space parametrized by $p_{1}$ and $q_{1}$. Notice, that the semi-classical approximation is exact in this case. This has to be compared with the computation of the Reshetikhin-Turaev-Witten invariants [3, 100] for torus knots in $\mathbf{S}^{3}$, where the stationary phase approximation is also exact, see e.g. [101.

24 Once again, we should remind that we tacitly omit the quotient by the Weyl group. 


\section{References}

[1] E. Witten, "Quantization Of Chern-Simons Gauge Theory With Complex Gauge Group," Commun. Math. Phys. 137, 29 (1991).

[2] C. Rovelli and L. Smolin, "Loop Space Representation Of Quantum General Relativity," Nucl. Phys. B 331, 80 (1990).

[3] E. Witten, "Quantum Field Theory And The Jones Polynomial," Commun. Math. Phys. 121, 351 (1989).

[4] D. Bar-Natan, "On the Vassiliev Knot Invariants," Topology 34 (1995) 423.

[5] M. Culler, P.B. Shalen, "Bounding separating incompressible surfaces in knot manifolds," Ann. Math. 117 (1983) 109.

[6] A. Achucarro and P. K. Townsend, "A Chern-Simons Action For Three-Dimensional Anti-De Sitter Supergravity Theories," Phys. Lett. B 180, 89 (1986).

[7] E. Witten, "(2+1)-Dimensional Gravity As An Exactly Soluble System," Nucl. Phys. B 311, 46 (1988).

[8] K. Ezawa, "Classical and quantum evolutions of the de Sitter and the anti-de Sitter universes in (2+1)-dimensions," Phys. Rev. D 49, 5211 (1994) [Addendum-ibid. D 50, 2935 (1994)].

[9] E. Buffenoir, K. Noui and P. Roche, "Hamiltonian quantization of Chern-Simons theory with SL $(2, C)$ group," Class. Quant. Grav. 19, 4953 (2002), arXiv:hepth/0202121].

[10] H.-J. Matschull, "On the relation between $2+1$ Einstein gravity and Chern Simons theory," Class. Quant. Grav. 16 (1999) 2599.

[11] I.M. Gelfand, R.A. Minlos, Z.Ya. Shapiro, "Representations of the Rotation and Lorentz Groups and Their Applications," New York, 1963.

[12] M.A. Naimark, "Linear Representations of the Lorentz Group," New York, 1964.

[13] S. Deser, R. Jackiw and G. 't Hooft, "Three-Dimensional Einstein Gravity: Dynamics Of Flat Space," Annals Phys. 152, 220 (1984).

[14] S. Deser and R. Jackiw, "Classical And Quantum Scattering On A Cone," Commun. Math. Phys. 118, 495 (1988).

[15] G. 't Hooft, "Nonperturbative Two Particle Scattering Amplitudes In (2+1) Dimensional Quantum Gravity," Commun. Math. Phys. 117, 685 (1988).

[16] E. Witten, "Topology Changing Amplitudes In (2+1)-Dimensional Gravity," Nucl. Phys. B 323, 113 (1989).

[17] S. Carlip, "Exact Quantum Scattering In (2+1)-Dimensional Gravity," Nucl. Phys. B 324, 106 (1989).

[18] P. de Sousa Gerbert, "On Spin And (Quantum) Gravity In (2+1)-Dimensions," Nucl. Phys. B 346, 440 (1990). 
[19] K. Koehler, F. Mansouri, C. Vaz and L. Witten, "Wilson Loop Observables In (2+1)Dimensional Chern-Simons Supergravity," Nucl. Phys. B 341, 167 (1990).

[20] J. B. Hartle and S. W. Hawking, "Wave Function Of The Universe," Phys. Rev. D 28, 2960 (1983).

[21] G. W. Gibbons and J. B. Hartle, "Real Tunneling Geometries And The Large Scale Topology Of The Universe," Phys. Rev. D 42, 2458 (1990).

[22] B. S. Dewitt, Phys. Rev. 160, 1113 (1967); J.A. Wheeler, in "Battelle Rencontres", Benjamin, New York, 1968.

[23] S. Carlip, "Notes on the (2+1)-dimensional Wheeler-DeWitt equation," Class. Quant. Grav. 11, 31 (1994).

[24] E. J. Martinec, "Soluble Systems In Quantum Gravity," Phys. Rev. D 30, 1198 (1984).

[25] V. Moncrief, "Reduction Of The Einstein Equations In (2+1)-Dimensions To A Hamiltonian System Over Teichmuller Space," J. Math. Phys. 30, 2907 (1989).

[26] A. Hosoya and K. i. Nakao, "(2+1)-Dimensional Pure Gravity For An Arbitrary Closed Initial Surface," Class. Quant. Grav. 7, 163 (1990).

[27] S. Carlip, "Observables, Gauge Invariance, And Time In (2+1)-Dimensional Quantum Gravity," Phys. Rev. D 42, 2647 (1990).

[28] S. Carlip, "Lectures on $(2+1)$ dimensional gravity," J. Korean Phys. Soc. 28, S447 (1995) arXiv:gr-qc/9503024.

[29] L. Rozansky, "A Contribution To The Trivial Connection To Jones Polynomial And Witten's Invariant Of 3-D Manifolds. 1," Commun. Math. Phys. 175 (1996) 275.

[30] K. Krasnov, "Holography and Riemann Surfaces," Adv. Theor. Math. Phys. 4 (2000) 929; "On Holomorphic Factorization in Asymptotically AdS 3D Gravity," hepth/0109198.

[31] W. Thurston, "Three-Dimensional Manifolds, Kleinian Groups and Hyperbolic Geometry," Bull. Amer. Math. Soc. (N.S.) 6 (1982) 357-381.

[32] G.D. Mostow, "Quasi-conformal mappings in n-space and the rigidity of hyperbolic space forms," Publ. IHES 34 (1968) 53.

[33] R. Meyerhoff, "The Chern-Simons Invariant of Hyperbolic 3-Manifolds," Thesis, Princeton University, 1981.

[34] R. M. Kashaev, "Quantum Dilogarithm as a 6j-Symbol," Mod. Phys. Lett. A9 (1994) 3757; R.M. Kashaev, "A Link Invariant from Quantum Dilogarithm," q-alg/9504020; R.M. Kashaev, "The hyperbolic volume of knots from quantum dilogarithm," $\mathrm{q}$ alg/9601025.

[35] H. Murakami, J. Murakami, "The colored Jones polynomials and the simplicial volume of a knot," math.GT/9905075.

[36] H. Murakami, J. Murakami, M. Okamoto, T. Takata, Y. Yokota, "Kashaev's conjecture and the Chern-Simons invariants of knots and links," math.GT/0203119. 
[37] N. Hayashi, "Quantum Hilbert space of G(C) Chern-Simons-Witten theory and gravity," Prog. Theor. Phys. Suppl. 114, 125 (1993).

[38] E. Witten, "Chern-Simons gauge theory as a string theory," Prog. Math. 133 (1995) 637, hep-th/9207094.

[39] R. Gopakumar, C. Vafa, "On the Gauge Theory/Geometry Correspondence," Adv. Theor. Math. Phys. 3 (1999) 1415.

[40] R. Gopakumar, C. Vafa, "M-theory and topological strings. I - II," hep-th/9809187, hep-th/9812127.

[41] G. Curio, "Superpotentials for M-theory on a G2 holonomy manifold and Triality symmetry," hep-th/0212211; "Superpotential of the M-theory conifold and type IIA string theory," hep-th/0212233.

[42] B. Acharya, "A Moduli Fixing Mechanism in M theory," hep-th/0212294.

[43] S. Elitzur, G. W. Moore, A. Schwimmer and N. Seiberg, "Remarks On The Canonical Quantization Of The Chern-Simons-Witten Theory," Nucl. Phys. B 326, 108 (1989).

[44] K. S. Thorne, R. H. Price and D. A. Macdonald, "Black Holes: The Membrane Paradigm," NEW HAVEN, USA: YALE UNIV. PR. (1986).

[45] L. Susskind, L. Thorlacius and J. Uglum, "The Stretched horizon and black hole complementarity," Phys. Rev. D 48, 3743 (1993).

[46] D. Cooper, M. Culler, H. Gillet, D.D. Long, P.B. Shalen, "Plane curves associated to character varieties of 3-manifolds," Invent. Math. 118 (1994) 47.

[47] K. Krasnov, "3D gravity, point particles and Liouville theory," Class. Quant. Grav. 18 (2001) 1291.

[48] N. Seiberg, E. Witten, "Monopole Condensation, And Confinement In N=2 Supersymmetric Yang-Mills Theory," Nucl.Phys. B426 (1994) 19; Erratum-ibid. B430 (1994) 485.

[49] S. Carlip and C. Teitelboim, "The Off-shell black hole," Class. Quant. Grav. 12, 1699 (1995), arXiv:gr-qc/9312002.

[50] S. Carlip and C. Teitelboim, "Aspects of black hole quantum mechanics and thermodynamics in (2+1)-dimensions," Phys. Rev. D 51, 622 (1995), [arXiv:gr-qc/9405070.

[51] S. Carlip, "The (2+1)-Dimensional Black Hole," Class.Quant.Grav. 12 (1995) 2853.

[52] W. Neumann, D. Zagier, "Volumes of Hyperbolic Three-Manifolds," Topology 24 (1985) 307.

[53] D. Cooper, D. Long, "Remarks on the A-polynomial of a Knot," J. Knot Theory and Its Ramifications, 5 (1996) 609.

[54] D. Cooper, D. Long, "Representation Theory and the A-polynomial of a Knot," Chaos, Solitons, and Fractals, 9 (1998) 749.

[55] T. Yoshida, "The $\eta$-invariant of hyperbolic 3-manifolds," Invent. Math. 81 (1985) 473. 
[56] H.M. Hilden, M.T. Lozano, J.M. Montesinos-Amilibia, "On Volumes and ChernSimons Invariants of Geometric 3-Manifolds," J. Math. Sci. Univ. Tokyo 3 (1996) 732.

[57] J. E. Nelson, T. Regge and F. Zertuche, "Homotopy Groups And (2+1)-Dimensional Quantum De Sitter Gravity," Nucl. Phys. B 339, 516 (1990).

[58] J.M.F. Labastida, M. Marino, C. Vafa, "Knots, links and branes at large N," JHEP 0011 (2000) 007.

[59] S. Axelrod, S. Della Pietra and E. Witten, "Geometric Quantization Of Chern-Simons Gauge Theory," J. Diff. Geom. 33, 787 (1991).

[60] N. J. Hitchin, "Flat Connections And Geometric Quantization," Commun. Math. Phys. 131, 347 (1990).

[61] J. Weitsman, "Quantization via Real Polarization of the Moduli Space of Flat Connections and Chern-Simons Gauge Theory in Genus One," Commun. Math. Phys. 137, 175 (1991); "Real Polarization of the Moduli Space of Flat Connections on a Riemann Surface," Commun. Math. Phys. 145, 425 (1992).

[62] N.M.J. Woodhouse, "Geometric Quantization," Oxford University Press, 1991.

[63] S. Bates, A. Weinstein, "Lectures on the Geometry of Quantization," Berkeley, 1997.

[64] C.D. Hodgson, "Degeneration and regeneration of geometric structures on threemanifolds," Thesis, Princeton University, 1986.

[65] N. Dunfield, "Cyclic surgery, degrees of maps of character curves, and volume rigidity of hyperbolic manifolds," Invent. Math. 136 (1999) 623.

[66] P. Kirk, E. Klassen, "Chern-Simons Invariants of 3-Manifolds Decomposed along Tori and the Circle Bundle over the Representation Space of $T^{2}$," Commun. Math. Phys. 153 (1993) 521.

[67] D. Bar-Natan and E. Witten, "Perturbative expansion of Chern-Simons theory with noncompact gauge group," Commun. Math. Phys. 141, 423 (1991).

[68] J.S. Birman, "New Points of View in Knot Theory," Bull. Amer. Math. Soc. 28 (1993) 253; J.S. Birman and X.S. Lin, "Knot Polynomials and Vassiliev Invariants," Invent. Math. 111 (1993) 225.

[69] S. Garoufalidis, "Difference and differential equations for the colored Jones function," math.GT/0306229; "On the characteristic and deformation varieties of a knot," math.GT/0306230.

[70] A. Casson, MSRI Lecture Notes, Berkeley (1985).

[71] C. Taubes, "Casson's Invariant and Gauge Theory, J. Diff. Geom. 31 (1990) 547.

[72] M.F. Atiyah, "The Geometry and Physics of Knots," Cambridge Univ. Press, 1990.

[73] A.A. Beilinson, V.G. Drinfeld, "Quantization of Hitchin's fibrations and Langlands' program," Math. Phys. Stud. 19, Kluwer Acad. Publ. (1996) 3.

[74] F. Bonahon, "A Schlafli-type formula for convex cores of hyperbolic 3-manifolds," J. Diff. Geom. 50 (1998) 24. 
[75] D. Thurston, "Hyperbolic Volume and the Jones Polynomial," talk presented at the Summer School in Grenoble, 1999.

[76] H. Murakami, "Optimistic calculations about the Witten-Reshetikhin-Turaev invariants of closed three-manifolds obtained from the figure-eight knot by integral Dehn surgeries," math.GT/0005289.

[77] S. Baseilhac and R. Benedetti, "Quantum Hyperbolic State Sum Invariants of 3Manifolds," math.GT/0101234.

[78] V.F.R. Jones, "A polynomial invariant for knots via von Newmann algebras," Bull. Amer. Math. Soc. 12 (1985) 103.

[79] R.M. Kashaev, O. Tirkkonen, "Proof of the volume conjecture for torus knots," math.GT/9912210.

[80] S. Baseilhac, R. Benedetti, "QHI, 3-manifolds scissors congruence classes and the volume conjecture," Geom. Topol. Monogr. 4 (2002) 13.

[81] Y. Yokota, "On the volume conjecture for hyperbolic knots," math.QA/0009165.

[82] K. Hikami, "Hyperbolic Structure Arising from a Knot Invariant," math-ph/0105039.

[83] Y. Akutsu, T. Deguchi, T. Ohtsuki, "Invariants of Colored Links," J. Knot Theory Ramif. 1 (1992) 161.

[84] R. K. Kaul and T. R. Govindarajan, "Three-dimensional Chern-Simons theory as a theory of knots and links," Nucl. Phys. B 380, 293 (1992), hep-th/9111063.

[85] H. Murakami "Mahler measure of the colored Jones polynomial and the volume conjecture," math.GT/0206249.

[86] C. Frohman, R. Gelca, W. Lofaro, The A-polynomial from the noncommutative viewpoint," math.QA/9812048; R. Gelca, "On the relation between the A-polynomial and the Jones polynomial," math.QA/0004158.

[87] L. Rozansky, "The Trivial Connection Contribution to Witten's Invariant and Finite Type Invariants of Rational Homology Spheres," q-alg/9503011.

[88] P. Melvin, H. Morton, "The Colored Jones Function," Commun. Math. Phys. 169 (1995) 501.

[89] D. Bar-Natan, S. Garoufalidis, "On the Melvin-Morton-Rozansky Conjecture," Invent. Math. 125 (1996) 103.

[90] L. Rozansky, "Higher Order Terms in the Melvin-Morton Expansion of the Colored Jones Polynomial," q-alg/9601009.

[91] L. Rozansky, "The Universal R-Matrix, Burau Representaion and the Melvin-Morton Expansion of the Colored Jones Polynomial," q-alg/9604005.

[92] D. Freed, R. Gompf, "Computer Calculation of Witten's 3-Manifold Invariants," Commun. Math. Phys. 141 (1991) 79.

[93] R. Lawrence, "Asymptotic Expansions of Witten-Reshetikhin-Turaev Invariants of Some Simple 3-Manifolds," J. Math. Phys. 36 (1995) 6106.

[94] J. Milnor, "A Duality Theorem for Reidemeister Torsion," Ann. Math. 76 (1962) 137. 
[95] V. Turaev, "Reidemeister Torsion in Knot Theory," Russ. Math. Surveys 41 (1986) 97.

[96] M. Banados, C. Teitelboim, J. Zanelli, "The Black Hole in Three Dimensional Space Time," Phys. Rev. Lett. 69 (1992) 1849; M. Banados, M. Henneaux, C. Teitelboim, J. Zanelli, "Geometry of the 2+1 Black Hole," Phys.Rev. D48 (1993) 1506.

[97] M. Bos and V. P. Nair, "U(1) Chern-Simons Theory And C = 1 Conformal Blocks," Phys. Lett. B 223 (1989) 61; "Coherent State Quantization Of Chern-Simons Theory," Int. J. Mod. Phys. A 5 (1990) 959.

[98] G. V. Dunne, R. Jackiw and C. A. Trugenberger, "Chern-Simons Theory In The Schrodinger Representation," Annals Phys. 194 (1989) 197.

[99] M. Manoliu, "Abelian Chern-Simons theory," J. Math. Phys. 39 (1998) 170; "Quantization of symplectic tori in a real polarization," dg-ga/9609012.

[100] N.Y. Reshetikhin, V.G. Turaev, "Invariants of 3-manifolds via link polynomials and quantum groups," Invent. Math. 103 (1991) 547.

[101] R. Lawrence, L. Rozansky, "Witten-Reshetikhin-Turaev Invariants of Seifert Manifolds," Commun. Math. Phys. 205 (1999) 287. 